\title{
Diversity of the moth fauna (Lepidoptera: Heterocera) of a wetland forest: A case study from Motovun forest, Istria, Croatia
}

\author{
TONI KOREN ${ }^{1}$ \\ KAJA VUKOTIĆ2 \\ MITJA ČRNE ${ }^{3}$ \\ ${ }^{1}$ Croatian Herpetological Society - Hyla, \\ Lipovac I. n. 7, 10000 Zagreb \\ ${ }^{2}$ Biodiva - Conservation Biologist Society, \\ Kettejeva 1,6000 Koper, Slovenia \\ ${ }^{3}$ Biodiva - Conservation Biologist Society, \\ Kettejeva 1, 6000 Koper, Slovenia

\section{Correspondence:} \\ Toni Koren \\ koren.toni1@gmail.com
}

Key words: floodplain forest, wetland moth species

Received January 16, 2015.

Revised April 10, 2015.

Accepted April 14, 2015.

\section{Abstract}

Background and Purpose: The Motovun forest located in the Mirna river valley, central Istria, Croatia is one of the last lowland floodplain forests remaining in the Mediterranean area.

Materials and Methods: Between 2011 and 2014 lepidopterological research was carried out on 14 sampling sites in the area of Motovun forest. The moth fauna was surveyed using standard light traps tents.

Results and Conclusions: Altogether 403 moth species were recorded in the area, of which 65 can be considered at least partially hygrophilous. These results list the Motovun forest as one of the best surveyed regions in Croatia in respect of the moth fauna. The current study is the first of its kind for the area and an important contribution to the knowledge of moth fauna of the Istria region, and also for Croatia in general.

\section{INTRODUCTION}

uring the past 150 years, over 300 papers concerning the moths and butterflies of Croatia have been published (e.g. 1, 2, 3, 4, 5, $6,7,8$ ). The majority of these papers contain information about one or a few families of butterflies and moths and in most cases deal with only a limited part of Croatia, such as Zagreb (8), Podravina (9) or Gorski Kotar (10). The only series of papers containing more comprehensive data about the butterflies and moths of Croatia were published by Stauder $(4,5,6,7)$, but even in this case, only the Adriatic coastline and islands were considered.

In the last decade the situation greatly improved for butterflies, as a large number of papers dealing with the local fauna have been published (e.g. 11, 12, 13). This also included the first checklist of butterflies of Croatia (14). However, data about the moth fauna still remain very limited, and only a few papers have been published containing recent and more comprehensive data (e.g. 15, 16, 17).

The main reasons for such limited knowledge has remained the same over time - a lack of continuity, financial support, and local experts for most moth groups. Many moth species are small, and not particularly brightly-coloured; distinguishing features are more difficult in comparison with butterflies, and the extraction of genitalia for correct identification is needed in many cases. In addition, many moth families still lack comprehensive field guides and identification keys, especially those likely to be present on the Balkan peninsula, making correct identification of species very difficult. Because of this, many of the most interest- 


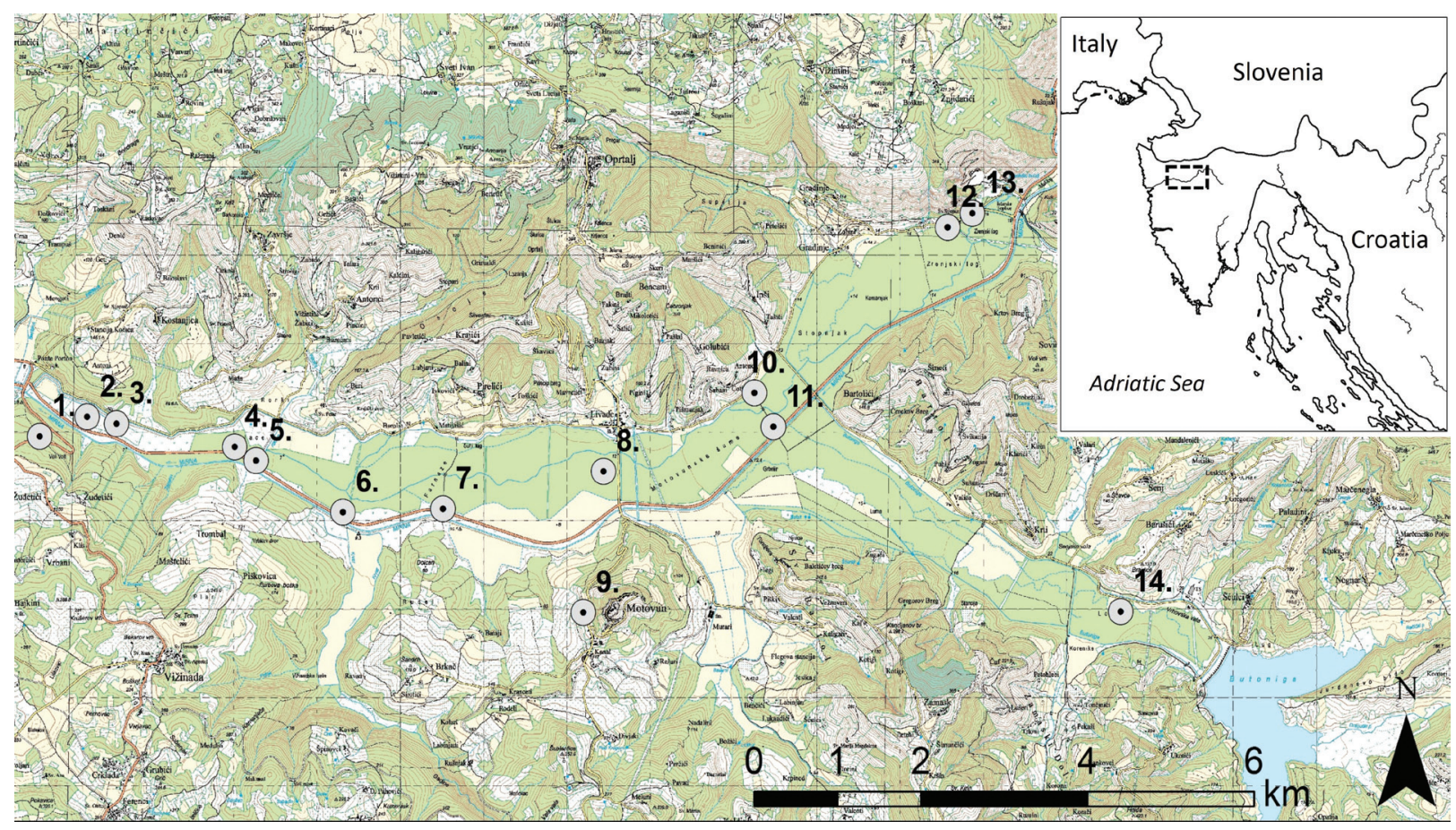

Figure 1. Map of Northern Adriatic with the location of the studied area and the studied localities inside the Motovun forest. Numbers correspond to the ones given in Table 1 .

ing ecological areas in Croatia have remained mostly or completely unsurveyed and their fauna unknown. Such localities include a range of important habitats, including marshes and moors, high mountains and riparian forests. Papers concerned with faunistics may be less popular than those dealing with complex taxonomic issues, but they nevertheless have an immense significance as they form the baseline of data upon which conservation is founded. In a country like Croatia, where very little is known about the fauna, such surveys are crucial.

Here we present the first systematic data about moths for a previously unsurveyed area, the Motovun forest.

\section{MATERIAL AND METHODS}

This study took place at 13 localities at the central part of the Motovun forest and one locality in the village Motovun (Fig. 1, Tab. 1) between 2011 and 2014. Each locality was visited from one to 13 times, during most of the vegetation seasons. On average, four hours were spent on each locality on each date, depending on the time of year and the climate conditions. One to three UV light tents were used per locality to attract moths. Most of the attracted moths were identified in the field and released. Only in cases where the identification was questionable were the moths collected and identified in the lab using standard identification keys (e.g. 18, 19, 20, 21, 22, 23, 24). Some members of the families Erebiidae, Geometridae and Noctuidae, all the encountered microlepidoptera were collected and stored in the private collection of the first author. The identification of all microlepidoptera and most of the collected macrolepidoptera was additionally checked by Stanislav Gomboc from Slovenia, on the basis of his extensive knowledge and the comparison with his validated moth collection containing most species of the northern Balkans. The nomenclature follows the online database Fauna Europae (25). For each species, larval host plants and habitat preference was given using standard literature $(18,19,20,21,22,23,24)$.

\section{Study area}

The Motovun forest is situated in the central part of the peninsula of Istria, in the alluvial valley of the Mirna River and its tributaries, southwest of Buzet and north of Motovun. In terms of vegetational and ecological contrasts Motovun differs greatly from other forest areas of the Istrian peninsula. The Mirna valley allows the influence of the sea to reach deep into the mainland part of the peninsula and the peculiarity of the landscape in the valley causes air stagnation and the occurrence of major climate extremes. The Mirna river basin is relatively poorly covered with forest vegetation, and is characterized by a multitude of torrential streams, which cause occasional flooding of the valley. The geological structure of the valley consists of limestone, marl and sandstones (26).

These relief, climate and hydrological conditions, particularly soil characteristics, have resulted in the creation of a unique Mirna valley lowland forest ecosystem. This forest community is dominated by common oak (Quercus robur L.) and ash (Fraxinus angustifolia Vahl). Other low- 
Table 1. List of surveyed localities with dates of visits, habitat and coordinates.

\begin{tabular}{|c|c|c|c|c|c|}
\hline Locality & Dates of visits & Habitat & GK5 X & GK5 Y & $\begin{array}{l}\text { Altitude } \\
\text { ( } \mathrm{m} \text { a.s.l.) }\end{array}$ \\
\hline $\begin{array}{l}\text { 1. Bartolići, } 500 \mathrm{~m} \text { east of } \\
\text { the village }\end{array}$ & $\begin{array}{l}\text { 20.3.2011, 6.6.2011, 9.3.2012, 16.3.2012, } \\
\text { 26.3.2012, 6.4.2012, 7.4.2012, 20.4.2012, } \\
\text { 27.5.2012, 6.6.2012, 14.6.2012, 22.7.2012, } \\
\text { 17.8.2012 }\end{array}$ & mixed deciduous forest & 5410478 & 5024063 & 24 \\
\hline $\begin{array}{l}\text { 2. Golubići, } 500 \mathrm{~m} \text { south- } \\
\text { east of the village }\end{array}$ & 17.3.2012, 18.3.2012, 26.3.2012, 7.4.2012 & mixed deciduous forest & 5410251 & 5024446 & 21 \\
\hline 3. Istarske Toplice & $\begin{array}{l}\text { 26.3.2012, 16.4.2012, 20.4.2012, 8.5.2012, } \\
\text { 11.5.2012, 13.5.201, 26.6.2012, 14.6.2012 }\end{array}$ & mixed deciduous forest & 5412867 & 5026476 & 25 \\
\hline $\begin{array}{l}\text { 4. Livade, } 500 \mathrm{~m} \text { southern } \\
\text { from the village }\end{array}$ & $\begin{array}{l}\text { 9.3.2012, 25.3.2012, 26.3.2012, 5.4.2012, } \\
6.4 .2012,17.5 .2012,26.5 .2012,14.8 .2012\end{array}$ & $\begin{array}{l}\text { mixed floodplain forest, forest } \\
\text { path }\end{array}$ & 5408438 & 5023558 & 19 \\
\hline 5. Motovun village & $\begin{array}{l}\text { 29.8.2011, 7.9.2011, 10.9.2011, 14.9.2011, } \\
\text { 15.9.2011, 17.9.2011 }\end{array}$ & public light in the village & 5408194 & 5021964 & 252 \\
\hline $\begin{array}{l}\text { 6. Pirelići, } 1 \mathrm{~km} \text { south from } \\
\text { the village }\end{array}$ & $9.3 .2012,6.4 .2012,16.4 .2012,22.4 .2012$ & mixed floodplain forest & 5406512 & 5023136 & 11 \\
\hline $\begin{array}{l}\text { 7. Ponte Porton, } 2 \mathrm{~km} \text { east } \\
\text { of the village }\end{array}$ & 5.6 .2012 & mixed floodplain forest & 5404002 & 5023813 & 9 \\
\hline $\begin{array}{l}\text { 8. Ponte Porton, } 1 \mathrm{~km} \text { east } \\
\text { of the village }\end{array}$ & $\begin{array}{l}\text { 17.4.2013, 14.5.2012, 10.7.2013, 7.8.2013, } \\
\text { 7.8.2013, 15.8.2012, 4.9.2012, 7.11.2013, } \\
\text { 11.3.2014 }\end{array}$ & $\begin{array}{l}\text { rudimentary forest remains, } \\
\text { many Prunus bushes and } \\
\text { grassland }\end{array}$ & 5404009 & 5023836 & 9 \\
\hline $\begin{array}{l}\text { 9. Ponte Porton, } 500 \mathrm{~m} \text { east } \\
\text { of the village }\end{array}$ & $\begin{array}{l}\text { 17.4.2013, 14.5.2012, 10.7.2013, 7.8.2013, } \\
\text { 7.8.2013, 15.8.2012, 4.9.2012, 7.11.2013, } \\
\text { 11.3.2014 }\end{array}$ & grasslands on the forest edge & 5402237 & 5024174 & 6 \\
\hline $\begin{array}{l}\text { 10. Ponte Porton, } 500 \mathrm{~m} \text { east } \\
\text { of the village }\end{array}$ & $\begin{array}{l}\text { 17.4.2013, 14.5.2012, 10.7.2013, 7.8.2013, } \\
\text { 7.8.2013, 15.8.2012, 4.9.2012, 7.11.2013, } \\
\text { 11.3.2014 }\end{array}$ & mixed floodplain forest & 5402590 & 5024098 & 5 \\
\hline $\begin{array}{l}\text { 11. Sv. Stjepan around the } \\
\text { village }\end{array}$ & 13.5 .2012 & mixed deciduous forest & 5412570 & 5026318 & 24 \\
\hline $\begin{array}{l}\text { 12. Trombal, } 1.5 \mathrm{~km} \text { north- } \\
\text { east of the village }\end{array}$ & $\begin{array}{l}\text { 9.3.2012, } 21.3 .2012,16.4 .2012,18.4 .2012, \\
17.5 .2012,18.5 .2012,27.5 .2012,20.6 .2012, \\
16.7 .2012,14.8 .2012,17.8 .2012,13.9 .2012\end{array}$ & $\begin{array}{l}\text { forest path surrounded by } \\
\text { mixed forest }\end{array}$ & 5405507 & 5023769 & 17 \\
\hline $\begin{array}{l}\text { 13. Valice-Krti, forest path } \\
\text { near the village }\end{array}$ & $\begin{array}{l}\text { 17.3.2012, 27.3.2011, 20.4.2012, 2.6.2012, } \\
\text { 6.6.2012, 22.7.2012, 17.8.2012 }\end{array}$ & $\begin{array}{l}\text { rudimentary forest remains, } \\
\text { many Prunus bushes }\end{array}$ & 5414646 & 5021973 & 25 \\
\hline $\begin{array}{l}\text { 14. Žudetići, } 400 \mathrm{~m} \text { south- } \\
\text { ern from the village }\end{array}$ & $\begin{array}{l}\text { 3.3.2012, 17.3.2012, } 21.3 .2012,26.3 .2012, \\
\text { 10.4.2012, 4.7.2012, 5.7.2012, 6.7.2012, } \\
\text { 22.7.2012, 9.9.2012, 8.10.2012 }\end{array}$ & forest path in a mixed forest & 5401667 & 5023955 & 29 \\
\hline
\end{tabular}

land forest trees like black alder (Alnus glutinosa Gaertn.), poplar (Populus alba L. and Populus nigra L.), white willow (Salix alba L.) and elm (Ulmus carpinifolia Gleditsch.) are also present. This forest belongs to the Querceto-Fraxinetum angustifoliae association (26). As in all lowland forests, vegetation composition depends on the micro relief, and availability of water which is why the Querceto-Fraxinetum angustifoliae association is not the only forest community in Motovun (26). The catchment area of the valley is covered by different stages of degraded common oak forests.

During the last century many anthropogenic changes have occurred in the area, including the building of a new road in the southern part of the forest and the excavation of a new riverbed. These actions had a tremendous negative impact on the water regime of the Motovun forest and its pristine condition (26). Prior to the excavation of the new river bed, the forest surface was 1274 ha. In 1967 the government decided that a part of the forest should be cleared for agriculture, excluding the protected 281 ha near Istarske Toplice, a special reserve of forest vegetation. Approximately 250 ha of the forest was cleared. Additionally, due to the construction of the accumulation lake Botonega, another 80 ha were cleared, which left only about 944 ha of forest (26).

\section{RESULTS}

During this research we gathered data for 403 moth species within the area of the Motovun forest. The Noctuidae are the best-represented group, with a total of 102 recorded species, followed by Geometridae with 96 species and Erebiidae with 49 recorded taxa. Of the larger microlepidoptera families, we recorded 33 species of Crambidae, 21 of Tortricidae and 13 of Pyralidae. A full species list 
for each family found in this study is given in Appendix I. Most of the recorded moth species were mesophilous $(\mathrm{N}=202)$, followed by xerothermophilous $(\mathrm{N}=76)$ and mesophilous-hygrophilous $(\mathrm{N}=62)$. A total of 19 species showed clear hygrophilous affiliation, while 65 species being at least partially hygrophilous.

\section{DISCUSSION}

The Motovun forest is one of the most interesting areas in Istria, in terms of vegetation and ecological conditions but it remains almost completely a mystery in terms of insect diversity. It is surprising that almost no published data exists for the region. Besides our data, less than ten published records exist for the wider area of the Mirna valley $(4,5,6,7)$ but not for the forest itself. This makes comparison with historical data impossible, but at the other hand makes our data crucial for any further study of the moth fauna of the area.

With almost no existing checklists, atlases, regional overviews and with rare exceptions $(9,15,16,17)$, few moth surveys carried out in the last 40 years, it is difficult to put our data into a meaningful context. In the wider area of the Kupa River, about 400 species were recorded during a long term survey $(27,28,29,30,31)$. On the other hand, a 30-year long study on Krk Island resulted in more than 1000 recorded species, although this also included all the microlepidoptera families (15). Both examples included a large variety of habitats and a much larger area than in this present study. The only recent survey of a wetland area is that of Kopački Rit, where 201 species were recorded during a year-long moth survey (16). That number probably represents only a fraction of the species inhabiting the area, and the authors themselves concluded that additional surveys are needed (16).

Compared with the mentioned results from the other regions of Croatia, the area of Motovun forest is now one of the best surveyed areas in Croatia. The number of moth species living in Croatia is unknown, but is hypothesised to be about 3000 (32). For the area of former Yugoslavia, a total of 3454 species have been recorded (33), however, this includes the territory of whole ex-Yugoslavia, and the real number of species recorded from Croatia is certainly lower. On the other hand, as this list is not up to date, and many species need to be added to the list, due to new species records as well as the description of new species, the number of species will be somewhat higher. This only implies the need for the detailed revision and the creation of the recent checklists of moths of Croatia.

During this preliminary survey more attention was given to larger moths, while Microlepidoptera were collected only occasionally, and thus are far less represented in this work and will be dealt with in future surveys. The same goes for some difficult genera of larger moths (e.g. the geometrid genus Eupithecia Curtis 1825).

These results are the first systematic survey of the Motovun forest, and they can be used to make decisions about the conservation and management of natural resources, especially for insect biodiversity, in the future. During our survey we recorded 19 strictly wetland species, of which the records of Chilo phragmitella (Hubner 1805), Acronicta strigosa (Denis \& Schiffermüller, 1775), Phragmataecia castaneae (Hübner, 1790) and Colobochyla salicalis (Denis \& Schiffermüller, 1775) are of most of the interest, as the species are very local in Croatia. Aside from the river Mirna, many small streams are present in the forest, in which many of the more common hostplants for wetland species can be observed. Due to that, it is more than probable that the species do develop in the area, but further combined entomological-floristic surveys of the area would for sure greatly contribute to the knowledge about the hostplants of wetland species in the area. As it is now, the ecological affiliation, as well as the hostplant list (Appendix I) was based only on the knowledge from other parts of Europe (e.g. 18, 19, 20, 21, 22, 23, 24) so it is possible that they are not fully correct and do not apply fully to the populations of the species living in Croatia. In any case, the presence of these species in the area indicate that the Mirna river valley still represents suitable habitat, and probably represents a refuge for wetland species in Istria. Aside from the Mirna river valley, only several other wetland habitats remain on the peninsula: two medium-sized rivers, Dragonja in the north and Raša in the east as well as the only swamp, Palud near Rovinj. None of these areas had been surveyed in the past, so no comparison with the Mirna river vally is possible. Hovever, it would be of great interest to survey the moth fauna of all the mentioned areas in the near future. Wetland moth communities are especially endangered, since such habitats were decimated during the last century, and are very rare nowadays. This is especially true in the karstic areas of Croatia, including Istria, the Adriatic islands and Dalmatia.

The Motovun river valley is also of a great importance for other animal groups. This is the area with the most records of the globally endangered False Ringlet Coenonympha oedippus (Fabricius, 1787), and the most southern distribution border for this species (34). How poorly this area was surveyed in the past is demonstrated by the fact that this species was recorded in Croatia less than two decades ago (34). Aside from invertebrates, the area is also interesting because of the vertebrate fauna. In the area, the strongest and largest population of the the Italian agile frog, Rana latastei Boulenger, 1879 in Croatia has been recorded (35). Our results show that the Motovun forest represents an area of high biodiversity. Most of the forest area is criss-crossed by roads, forest paths and macadam roads, but except from the main road leading from Buzet to Ponte Porton, most of other roads are with very little traffic. For the conservation of the moth fauna of the area, the most important action is to conserve the current forest limits and to avoid any alteration to the finely-balanced hydrological regime.

During this study, the sampling period was sometimes very brief (e.g. two hours) during the single nights, due to 
unfavourable weather conditions like low temperatures or high humidity in the air. In such conditions moth activity is very limited, and most of the species do not fly at all, so the moth observation was ceased earlier. To compensate this, we visited the same locality longer during the next visits. In order to gain additional knowledge about the species diversity of the Motovun forest, additional long term surveys are needed. An increase in the amount of UV light traps would be especially beneficial for such studies, since many more species are attracted if the number of light traps is greater. Additionally, more different habitats and locations should be surveyed to gain a more complete insight into the moth diversity of the region.

\section{Acknowledgments}

We are grateful to all colleagues who helped us with the field work, as well as to Stanislav Gomboc who helped us in the identification of some moth species.

\section{REFERENCES}

1. MANN J 1857 Verzeichniss der im Jahre 1853 in der Gegend von Fiume gesammelten Schmetterlinge. Wiener entomologische Monatschrift 1 (6): 139-189

2. MANN J 1867 Schmetterlinge gesammelt im J. 1866 um Josefsthal in der croat. Militärgrenze. Verhandlunden zoologisch-botanischen Gesellschaft in Wien 17: 63-76

3. MANN J 1869 Lepidopteren gesammmelt während dreier reisen nach Dalmatien in den Jahren 1850, 1862 und 1868. Verhandlunden zoologisch-botanischen Gesellschaft in Wien 19: 371-388

4. STAUDER H 1923 Die Schmetterlingsfauna der illyro-adriatischen Festland-und Inselzone (Faunula Illyro-Adriatica). Zeitschrift für wissenschaftliche Insektenbiologie 18: 10-18, 58-68, 106-114, 187202, 256-267, 317-327 [The butterflies of the Illyro-Adriatic, mainland and the islands]

5. STAUDER H 1925 Die Schmetterlingsfauna der illyro-adriatischen Festland-und Inselzone. (Faunula-Illyro-Adriatica). Zeitschrift für wissenschaftliche Insektenbiologie 20: 191-226 [The butterflies of the Illyro-Adriatic, mainland and the islands]

6. STAUDER H 1926 Die Schmetterlingsfauna der illyroadriatischen Festland-und Inselzone (Faunula Illvro-Adriatica). Zeitschrift für wissenschaftliche Insektenbiologie 21: 179-190, 223-238 [The butterflies of the Illyro-Adriatic, mainland and the islands]

7. STAUDER H 1927 Die Schmetterlingsfauna der illyro-adriatischen Festland-und Inselzone. (Faunula Illyro-Adriatica). Zeitschrift für wissenschaftliche Insektenbiologie 22: 30-45, 74-92 [The butterflies of the Illyro-Adriatic, mainland and the islands]

8. MLADINOV L 1958 Popis noćnih leptira (Noctua) Zagreba i okolice. Hrvatski narodni zoološki muzej 1, Zagreb, p 61 [List of moths (Noctua) of Zagreb and its surroundings]

9. KRANJČEV R 1985 Odnos faune makrolepidoptera prema prirodnim i antropogenim staništima Podravine i podravskih pijesaka I. Podravski zbornik 11: 200-226 [Relationships of the macrolepidoptera fauna and the natural and anthropogenic habitats in Podravina and podravina sands]

10. KUČINIĆ M, IGALFFY K, ŠAŠIĆ, M, BALEN S 1994 A contribution of the Heterocera fauna (Insecta, Lepidoptera) of the centralmountain part (Risnjnak \& Lička Plješevica) of the Republic of Croatia. Natura Croatica 3 (1): 23-40

11. MIHOCI I, HRŠAK V, KUČINIĆ M, MIČETIĆ STANKOVIĆ V, DELIĆ A, TVRTKOVIĆ N 2011 Butterfly diversity and biogeography on the Croatian karst mountain Biokovo: Vertical distribution and preference for altitude and aspect? European Journal of
Entomology 108 (4): 623-633 http://dx.doi.org/10.14411/ eje.2011.081

12. VEROVNIK R 2011 Butterflies (Lepidoptera: Rhopalocera) of the Croatian islands: an update on published records and new surveys of Pašman and Ugljan. Entomologist's Gazette 62: 251-263

13. WITHRINGTON D K J, VEROVNIK R 2008 Butterflies (Rhopalocera) of the Croatian Islands. Entomologist's Gazette 59: 3-25

14. ŠAŠIĆ M, MIHOCI I 2011 Annotated checklist of Croatian butterflies with vernacular names. Natura Croatica 20: 425-436

15. HABELER H 2003 Die Schmetterlinge der Adria -insel Krk. Buchreihe zur Entomologie Esperiana, Graz, p 221

16. VIGNJEVIĆ G, ZAHIROVIĆ Ž, TURIĆ N, MERDIČ E 2010 Moths (Lepidoptera: Heterocera) of Kopački rit Nature Park - Results of preliminary research. Entomologica Croatica 14 (3-4): 17-32 [The butterflies of the Adriatic island Krk]

17. KOREN T, LADAVAC L 2013 Diversity of Macroheterocera (except fam. Noctuidae [sensu novo] and fam. Geometridae) in central Istria. Natura Croatica 22 (1): 73-94

18. FIBIGER M 1993 Noctuinae II. Noctuidae Europaeae. Volume 2. Entomological Press, Soros, p 230

19. FIBIGER M 1997 Noctuidae Europaeae. Vol. 3 (Noctuinae III). Entomological Press, Soros, p 418

20. LERAUT P 2006 Moths of Europe, Volume 1: Saturnids, Lasiocampids, Hawkmoths, Tiger Moths. NAP Editions, Verrières-le Buisson, $\mathrm{p} 400$

21. LERAUT P 2009 Moths of Europe, Volume 2: Geometrid Moths. NAP Editions, Verrières-le Buisson, p 808

22. LERAUT P 2012 Moths of Europe, Volume 3: Zygaenids, Pyralids 1 and Brachodids. NAP Editions, Verrières-le Buisson, p 600

23. LERAUT P 2014 Moths of Europe, Volume 4: Pyralids 2. NAP Editions, Verrières-le Buisson, p 440

24. NOWACKI J 1998 The Noctuids (Lepidoptera: Noctuidae) of Central Europe. Coronet Books, Bratislava, p 51

25. KARSHOLT O, VAN NIEUKERKEN E J (eds.) 2011 Lepidoptera. Fauna Europaea version 2.4. Web Service available online at http://www.faunaeur.org.

26. PRPIĆ B 1980 Problematika Motovunske šume s prijedlogom rješenja. Šumarski list 1980: 5-6 [Problematics of the Motovun forest with the examples of the solutions]

27. MLADINOV L 1976 Lepidoptera iz doline gornjeg toka rijeke Kupe. II. Bombyces i Sphings. Acta Entomologica Jugoslavica 12(12): 89-98 [Lepidoptera from the lower part of Kupa river. II. Bombycidae \& Sphingidae]

28. MLADINOV L 1977 Lepidoptera iz doline gornjeg toka rijeke Kupe III. Noctuidae. Acta Entomologica Jugoslavica 13: 77-88 [Lepidoptera from the lower part of Kupa river. III. Noctuidae]

29. MLADINOV L 1978 Prvi dodatak poznavanju faune Macrolepidoptera iz doline gornjeg toka rijeke Kupe. Acta Entomologica Jugoslavica. 14(1-2): 63-67 [The first addition to the Macrolepidoptera fauna from the lower part of Kupa river]

30. MLADINOV L 1980 Lepidoptera iz doline gornjeg toka rijeke Kupe IV. Geometridae. Acta Entomologica Jugoslavica 16: 69-91 [Lepidoptera from the lower part of Kupa river. IV. Geometridae]

31. MLADINOV L 1983 Drugi dodatak poznavanju faune Macrolepidoptera iz doline gornjeg toka rijeke Kupe. Acta Entomologica Jugoslavica 19 (1-2): 47-51[The second addition to the Macrolepidoptera fauna from the lower part of Kupa river]

32. KUČINIĆ M, PLAVAC I 2009 Danji leptiri. Priručnik za inventarizaciju i praćenje stanja. Državni zavod za zaštitu prirode, Zagreb, p 4 [Butterflies. Handbook for inventorying and monitoring of the status]

33. KARSHOLT O, RAZOWSKI J 1996 The Lepidoptera of Europe: A distributional Checklist. Apollo Books.

34. KUČINIĆ M, TVRTKOVIĆ N, KLETEČKI E 1999 The False Ringelt (Coenonympha oedippus F.) is a member of the Croatian butterfly fauna after all. Natura Croatica 8 (4):399-405

35. KULJERIĆM 2011 Italian agile frog, Rana latastei Boulenger, 1879 (Amphibia, Anura). HYLA - Herpetološki bilten 2011 (2): 3-20 


\section{APPENDIX I. \\ SYSTEMATIC LIST OF RECORDED MOTH SPECIES, THEIR HABITAT PREFERENCES, HOST PLANTS AND LOCALITIES ON WHICH THEY WERE RECORDED. NUMBERS CORRESPOND TO THE LOCALITIES IN TAB. 1.}

\begin{tabular}{|c|c|c|c|}
\hline Systematic list of recorded species & Habitat preference & Host plants & Locality \\
\hline \multicolumn{4}{|l|}{ Adelidae } \\
\hline 1. Adela reaumurella (Linnaeus 1758) & mesophilous & Quercus, Betula & 6. \\
\hline 2. $\quad$ Nemophora degeerella (Linnaeus 1758) & hygrophilous & Anemone nemorosa & 6. \\
\hline 3. Nematopogon swammerdamella (Linnaeus 1758) & mesophilous & dead leafs & 1. \\
\hline \multicolumn{4}{|l|}{ Blastobasidae } \\
\hline 4. $\quad$ Blastobasis glandulella (Riley 1871) & mesophilous & Quercus & 8. \\
\hline \multicolumn{4}{|l|}{ Chimbachidae } \\
\hline 5. Diurnea fagella (Denis \& Schiffermüller 1775) & mesophilous-hygrophilous & Vaccinium vitis-idaea, Salix repens & 8., 10. \\
\hline \multicolumn{4}{|l|}{ Cossidae } \\
\hline 6. Cossus cossus (Linnaeus 1758) & mesophilous & Salix, Populus tremula, Betula, Alnus, Malus & 6. \\
\hline 7. Dyspessa ulula (Borkhausen, 1790) & xerothermophilous & Allium flavum, Allium sativum & 1., 3., 5., 6., 9 . \\
\hline 8. $\quad$ Phragmataecia castaneae (Hübner 1790) & hygrophilous & Phragmites comunis, Phragmites australis & 3. \\
\hline 9. Zeuzera pyrina (Linnaeus 1761) & mesophilous & $\begin{array}{l}\text { Salix, Prunus spinosa, Prunus domestica, Prunus avium, } \\
\text { Malus, Crataegus }\end{array}$ & $2 ., 3$. \\
\hline \multicolumn{4}{|l|}{ Crambidae } \\
\hline 10. Elophila nymphaeata (Linnaeus 1758) & hygrophilous & Potamogeton, Nymphoides, Nymphaea, Sparganium & 14. \\
\hline 11. Nymphula nitidulata (Hufnagel 1767) & hygrophilous & Sparganium & 14. \\
\hline 12. Parapoynx stratiotata (Linnaeus 1758) & hygrophilous & Potamogeton, Nymphaea, Ceratophyllum & 3., 11., 14. \\
\hline 13. Agriphila geniculea (Haworth 1811) & xerothermophilous & Gramineae & $9 ., 13$. \\
\hline 14. Catoptria falsella (Denis \& Schiffermüller 1775) & mesophilous & $\begin{array}{l}\text { Barbula muralis, Trtula intermedia, T. muralis, Brach- } \\
\text { ythecium rutabulum }\end{array}$ & 3. \\
\hline 15. Catoptria pinella (Linnaeus 1758) & mesophilous & Deschampsia cespitosa, Cyperaceae, Bryum & 14. \\
\hline 16. Chilo phragmitella (Hubner 1805) & hygrophilous & Phragmites communis, Glyceria & $4 ., 14$. \\
\hline 17. Crambus lathoniellus (Zincken 1817) & mesophilous-hygrophilous & Deschampsia cespitosa, Aira & $5 ., 14$. \\
\hline 18. Pediasia contaminella (Hübner 1796) & xerothermophilous & Gramineae & 3. \\
\hline 19. Anania coronata (Hufnagel 1767) & mesophilous & $\begin{array}{l}\text { Sambucus nigra, Syringa vulgaris, Convolvulus arvensis, } \\
\text { Fraxinus, Ligustrum vulgare, Viburnum }\end{array}$ & $3 ., 6$. \\
\hline 20. Anania lancealis (Denis \& Schiffermüller 1775) & mesophilous-hygrophilous & Eupatorium cannabinum, Stachys, Senecio & $6 ., 12$. \\
\hline 21. Anania stachydalis (Germar 1821) & mesophilous & Stachys, Stachys sylvatica & 14. \\
\hline 22. Loxostege sticticalis (Linnaeus 1761) & xerothermophilous & Artemisia vulgaris, Atriplex & 3. \\
\hline 23. Nascia cilialis (Hubner 1796) & hygrophilous & Carex, Cladium mariscus & $2 ., 5$. \\
\hline 24. Ostrinia nubilalis (Hübner 1796) & xerothermophilous & Artemisia vulgaris, Rumex, Malva, Humulus lupulus & 3., $11 ., 14$. \\
\hline 25. Pyrausta aurata (Scopoli 1763) & $\begin{array}{l}\text { mesophilous-xerother- } \\
\text { mophilous }\end{array}$ & $\begin{array}{l}\text { Origanum vulgare, Mentha aquatica, Mentha arvensis, } \\
\text { Thymus Salvia }\end{array}$ & 9. \\
\hline 26. Pyrausta despicata (Scopoli 1763) & mesophilous & Plantago, Salvia, Gnaphalium & $\begin{array}{l}1 ., 3 ., 6 ., 8 . \\
10 ., 14 .\end{array}$ \\
\hline 27. Pyrausta purpuralis (Linnaeus 1758) & $\begin{array}{l}\text { mesophilous-xerother- } \\
\text { mophilous }\end{array}$ & $\begin{array}{l}\text { Mentha aquatica, Mentha arvensis, Origanum vulgare, } \\
\text { Prunella vulgaris, Thymus }\end{array}$ & 14. \\
\hline 28. Pyrausta rectefascialis Toll 1936 & mesophilous & Salvia, Thymus & 3. \\
\hline 29. Sitochroa verticalis (Linnaeus 1758) & mesophilous & $\begin{array}{l}\text { Cirsium arvense, Atriplex, Rumex, Urtica, Centaurea, } \\
\text { Viola odorata, Cytisus scoparius }\end{array}$ & 14. \\
\hline 30. Uresiphita gilvata (Fabricius 1794) & mesophilous & Genista, Ulex, Cytisus, Chamaecytisus & 3. \\
\hline 31. Sclerocona acutella (Eversmann 1842) & hygrophilous & Phragmites australis & 3., $5 ., 14$. \\
\hline 32. Eudonia delunella (Stainton 1849) & mesophilous & lichens & $5 ., 14$. \\
\hline 33. Eudonia mercurella (Linnaeus 1758) & mesophilous & Hypnum, Bryophyta & 3., 6., 8., 11 . \\
\hline 34. Eudonia pallida (Curtis 1827) & mesophilous & lichens, Bryophyta & 5. \\
\hline
\end{tabular}




\begin{tabular}{|c|c|c|c|}
\hline Systematic list of recorded species & Habitat preference & Host plants & Locality \\
\hline 35. Scoparia basistrigalis Knaggs 1866 & mesophilous & lichens & 13. \\
\hline 36. Scoparia perplexella (Zeller 1839) & mesophilous & unknown & 12. \\
\hline 37. Scoparia pyralella (Denis \& Schiffermüller 1775) & mesophilous & Senecio jacobaea & 6. \\
\hline 38. Agrotera nemoralis (Scopoli 1763) & mesophilous & Carpinus betulus, Castanea sativa, Quercus & $6 ., 11 ., 13 ., 14$. \\
\hline $\begin{array}{l}\text { 39. Dolicharthria punctalis (Denis \& Schiffermüller } \\
1775 \text { ) }\end{array}$ & xerothermophilous & $\begin{array}{l}\text { Centaurea, Plantago, Trifolium, Artemisia vulgaris, } \\
\text { Lotus }\end{array}$ & $2 ., 3$. \\
\hline 40. Metasia ophialis (Treitschke 1829) & mesophilous & unknown & 14. \\
\hline 41. Palpita vitrealis (Rossi 1794) & xerothermophilous & $\begin{array}{l}\text { Jasminum officinale, Olea europaea, Ligustrum, Fraxi- } \\
\text { nus, Arbutus unedo }\end{array}$ & 8. \\
\hline 42. Pleuroptya ruralis (Scopoli 1763) & mesophilous & $\begin{array}{l}\text { Urtica dioica, Chenopodium, Atriplex, Humulus lupulus, } \\
\text { Filipendula ulmaria, Ribes, Spiraea }\end{array}$ & 6., 13., 14. \\
\hline 43. Udea ferrugalis (Hubner 1796) & mesophilous & Stachys, Eupatorium, Arctium, Mentha, Fragaria & 1., 2., $6 ., 14$. \\
\hline \multicolumn{4}{|l|}{ Drepanidae } \\
\hline 44. Cilix glaucata (Scopoli 1763) & xerothermophilous & $\begin{array}{l}\text { Prunus spinosa, Prunus domestica, Crataegus, Malus } \\
\text { sylvestris }\end{array}$ & 6. \\
\hline 45. Watsonalla binaria (Hufnagel 1767)* & mesophilous & Quercus & $6 ., 8$. \\
\hline 46. Asphalia ruficollis (Denis \& Schiffermuller 1775) & xerothermophilous & Quercus & $6 ., 7$. \\
\hline 47. Cymatophorina diluta (Denis \& Schiffermuller 1775) & mesophilous & Quercus & 7. \\
\hline 48. Habrosyne pyritoides (Hufnagel 1766) & mesophilous & Rubus plicatus, Rubus & 6. \\
\hline 49. Polyploca ridens (Fabricius 1787) & xerothermophilous & Quercus & $1 ., 14$. \\
\hline 50. Tethea ocularis (Linnaeus 1767) & mesophilous & Populus tremula & $6 ., 8$. \\
\hline 51. Tethea or (Denis \& Schiffermüller 1775) & mesophilous & Populus tremula & $3 ., 6$. \\
\hline 52. Thyatira batis (Linnaeus 1758) & mesophilous & Rubus, Rubus plicatus & $\begin{array}{l}\text { 3., 6., 12., 13., } \\
14 .\end{array}$ \\
\hline \multicolumn{4}{|l|}{ Elachistidae } \\
\hline 53. Agonopterix yeatiana (Fabricius 1781) & mesophilous & Daucus carota, Oenanthe & 14. \\
\hline 54. Ethmia bipunctella (Fabricius 1775) & xerothermophilous & $\begin{array}{l}\text { Echium vulgare, Symphytum, Cynoglossum, Anchusa, } \\
\text { Lithospermum }\end{array}$ & 3. \\
\hline 55. Ethmia haemorrhoidella (Eversmann 1844) & mesophilous & unknown & 14. \\
\hline \multicolumn{4}{|l|}{ Erebidae } \\
\hline 56. Arctia villica (Linnaeus 1758) & xerothermophilous & $\begin{array}{l}\text { Taraxacum officinale, Plantago, Achillea millefolium } \\
\text { Lamium, Urtica, Centaurea, Fragaria, Leontodo }\end{array}$ & 2., 3., 9., 13., 14. \\
\hline 57. Diacrisia sannio (Linnaeus, 1758) & mesophilous & $\begin{array}{l}\text { Galium, Plantago, Urtica dioica, Alnus, Salix, Poly- } \\
\text { gonum, Trifolium, Calluna, vulgaris Cirsium, Taraxacum } \\
\text { officinale, Hieracium, Leontodon }\end{array}$ & $4 ., 6 ., 9$. \\
\hline 58. Euplagia quadripunctaria (Poda 1761) & mesophilous & $\begin{array}{l}\text { Lamium, Epilobium, Corylus, Rubus, Lonicera, Cytisus, } \\
\text { Urtica dioica }\end{array}$ & 14. \\
\hline 59. Phragmatobia fuliginosa (Linnaeus 1758) & mesophilous & Urtica, Lamium, Leontodon & 2., 3., 6., 14 . \\
\hline 60. Spilosoma lubricipeda (Linnaeus 1758) & mesophilous & $\begin{array}{l}\text { Urtica, Polygonum, Rumex, Rubus, Trifolium, Geranium, } \\
\text { Plantago, Taraxacum, officinale, Lactuca serriola, Rubus } \\
\text { plicatus }\end{array}$ & 6., $8 ., 13$. \\
\hline 61. Spilosoma lutea (Hufnagel 1766) & mesophilous & Urtica, Rumex, Plantago, Taraxacum officinale & 3., 6., 11., 13. \\
\hline 62. Eilema caniola (Hübner 1808) & xerothermophilous & lichens & 2., 4., 9., 14 . \\
\hline 63. Eilema complana (Linnaeus 1758) & mesophilous & lichens & $11 ., 14$. \\
\hline 64. Eilema depressa (Esper 1787) & mesophilous & lichens, Parmelia & 2., 3., 9., 13., 14. \\
\hline 65. Eilema sororcula (Hufnagel 1766) & mesophilous & lichens & $\begin{array}{l}3 ., 4 ., 6 ., 7 ., 10 . \\
11 ., 14 .\end{array}$ \\
\hline 66. Lithosia quadra (Linnaeus 1758) & mesophilous & lichens & 3., $6 ., 13$. \\
\hline 67. Miltochrista miniata (Forster 1771) & mesophilous & lichens & $1 ., 4 ., 6 ., 14$. \\
\hline 68. Pelosia muscerda Hufnagel 1766 & hygrophilous & lichens, Parmelia & 3., 5., 6., 11., 14. \\
\hline 69. Amata phegea (Linnaeus, 1758) & mesophilous & $\begin{array}{l}\text { Plantago, Rumex, Galium, Taraxacum officinale, Lam- } \\
\text { ium, Leontodon Lactuca }\end{array}$ & 2., 4., 9., 14. \\
\hline 70. Dysauxes ancilla (Linnaeus 1767) & xerothermophilous & Taraxacum, Senecio, Plantago, Lactuca & 3. \\
\hline 71. Dysauxes famula (Freyer 1836) & xerothermophilous & Taraxacum, Senecio, Plantago, Lactuca & 13. \\
\hline
\end{tabular}




\begin{tabular}{|c|c|c|c|}
\hline Systematic list of recorded species & Habitat preference & Host plants & Locality \\
\hline 72. Calyptra thalictri (Borkhausen 1790) & mesophilous & Thalictrum & 2., $3 ., 6$. \\
\hline 73. Catocala electa (Vieweg 1790) & hygrophilous & Salix & 1. \\
\hline 74. Catocala fulminea (Scopoli 1763) & $\begin{array}{l}\text { mesophilous-xerother- } \\
\text { mophilous }\end{array}$ & Prunus spinosa, Prunus domestica, Prunus padus & $1 ., 6$. \\
\hline 75. Catocala nymphagoga (Esper 1787) & xerothermophilous & Quercus & 1. \\
\hline 76. Catocala sponsa (Linnaeus 1767) & mesophilous & Quercus & 1. \\
\hline 77. Catephia alchymista (Denis \& Schiffermüller 1775) & mesophilous & Quercus & 3., \\
\hline 78. Dysgonia algira (Linnaeus 1767) & xerothermophilous & $\begin{array}{l}\text { Rubus, Salix, Genista, Parietaria officinalis, Ricinus com- } \\
\text { munis, Lythrum salicaria }\end{array}$ & $9 ., 14$. \\
\hline 79. Minucia lunaris (Denis \& Schiffermüller 1775) & $\begin{array}{l}\text { mesophilous-xerother- } \\
\text { mophilous }\end{array}$ & Quercus & 3. \\
\hline 80. Lygephila pastinum (Treitschke 1826) & mesophilous & Astragalus, Coronilla, Vicia & 2., 3., 14. \\
\hline 81. Lygephila procax (Hübner 1813) & xerothermophilous & Vicia, Coronilla, Lathyrus, Colutea & 11. \\
\hline 82. Eublemma purpurina (Denis \& Schiffermüller, 1775) & xerothermophilous & Cirsium arvense, Dictamnus albus & $5 ., 6$. \\
\hline 83. Eublemma ostrina (Hübner, 1808) & xerothermophilous & $\begin{array}{l}\text { Carduus, Carlina vulgaris, Echinops exaltatus, Cirsium, } \\
\text { Helichrysum arenarium }\end{array}$ & 3. \\
\hline 84. Herminia grisealis Denis \& Schiffermüller 1775 & mesophilous & $\begin{array}{l}\text { Quercus, Carpinus, Betula, Prunus, Crataegus, Alnus, } \\
\text { Urtica }\end{array}$ & 11. \\
\hline 85. Herminia tarsicrinalis (Knoch 1782) & mesophilous & Rubus, Clematis, Urtica & $\begin{array}{l}\text { 3., 4., 5., 6., } \\
13 ., 14 .\end{array}$ \\
\hline 86. Herminia tarsipennalis (Treitschke 1835) & mesophilous & dead leafs & $\begin{array}{l}\text { 1., 3., 5., 6., } \\
12 ., 13 .\end{array}$ \\
\hline 87. Herminia tenuialis (Rebel 1899) & xerothermophilous & Graminae & 2., 3., \\
\hline 88. Paracolax tristalis (Fabricius 1794) & mesophilous & dead leafs & 1., 6., 11., 14. \\
\hline 89. Pechipogo plumigeralis Hübner 1825 & xerothermophilous & Rubus, Cytisus, Rosa, Chamaecytisus, Hedera helix & 3., 13. \\
\hline 90. Pechipogo strigilata (Linnaeus 1758) & mesophilous & dead leafs & $2 ., 6$. \\
\hline 91. Polypogon tentacularia (Linnaeus 1758) & mesophilous-hygrophilous & Hieracium, Taraxacum officinale, Epilobium, Solidago & 3., 14. \\
\hline 92. Idia calvaria (Denis \& Schiffermüller, 1775) & hygrotermophilous & Populus, Salix & $5 ., 6$. \\
\hline 93. Zanclognatha lunalis (Scopoli 1763) & $\begin{array}{l}\text { mesophilous-xerother- } \\
\text { mophilous }\end{array}$ & dead leafs & 11., 14. \\
\hline 94. Hypena proboscidalis (Linnaeus 1758) & mesophilous & $\begin{array}{l}\text { Urtica dioica, Lamium, Humulus lupulus, Plantago, } \\
\text { Stachys, Aegopodium }\end{array}$ & 3. \\
\hline 95. Zekelita antiqualis (Hübner 1809) & xerothermophilous & Salvia officinalis & 1., 3., 11. \\
\hline 96. Colobochyla salicalis (Denis \& Schiffermüller 1775) & mesophilous-hygrophilous & Populus tremula, Salix caprea & 14. \\
\hline 97. Phytometra viridaria (Clerck 1759) & mesophilous-hygrophilous & Polygala vulgaris, Polygala serpyllifolia & 1., $11 ., 14$. \\
\hline 98. Trisateles emortualis (Denis \& Schiffermüller 1775) & mesophilous & Quercus, Fagus, Carpinus, Rubus & $6 ., 11 ., 14$. \\
\hline 99. Rivula sericealis (Scopoli 1763) & mesophilous-hygrophilous & Gramineae, Brachypodium, Carex, Poa & 3., 6., 13., 14. \\
\hline 100. Zebeeba falsalis (Herrich-Schäffer 1839) & xerothermophilous & Asparagus & 1., $12 ., 13$. \\
\hline 101. Lymantria dispar (Linnaeus 1758) & mesophilous & $\begin{array}{l}\text { Quercus, Arbores fructiferae, Salix, Populus, Crataegus, } \\
\text { Malus, Ulmus }\end{array}$ & 1., 2., 3., 6., 14 . \\
\hline 102. Lymantria monacha (Linnaeus 1758 ) & mesophilous & Pinus, Picea, Abies alba & 6. \\
\hline 103. Euproctis chrysorrhoea (Linnaeus 1758) & mesophilous & $\begin{array}{l}\text { Quercus, Arbores fructiferae, Prunus cerasus, Prunus, } \\
\text { Malus, Pyrus }\end{array}$ & 6., 13., 14. \\
\hline 104. Calliteara pudibunda (Linnaeus 1758) & mesophilous & Fagus, Betula, Quercus, Carpinus, Corylus & 7., 8. \\
\hline 105. Orgyia antiqua (Linnaeus 1758) & mesophilous & Salix, Sorbus aucuparia, Quercus, Prunus, Rosa canina & 1., 3., 11. \\
\hline \multicolumn{4}{|l|}{ Gelechiidae } \\
\hline 106. Dichomeris derasella (Denis \& Schiffermüller 1775) & mesophilous & $\begin{array}{l}\text { Crataegus, Malus sylvestris, Prunus cerasus, Prunus } \\
\text { spinosa Rubus caesius, Rubus fruticosus }\end{array}$ & 6., 11., $12 ., 14$. \\
\hline 107. Dichomeris ustalella (Fabricius 1794) & mesophilous & Tilia cordata & 13. \\
\hline \multicolumn{4}{|l|}{ Geometridae } \\
\hline 108. Alsophila aescularia (Denis \& Schiffermüller 1775) & mesophilous & $\begin{array}{l}\text { Quercus, Arbores fructiferae, Crataegus, Corylus avel- } \\
\text { lana, Prunus spinosa }\end{array}$ & $\begin{array}{l}\text { 1., 7., 8., } 10 ., \\
11 ., 14 .\end{array}$ \\
\hline 109. Abraxas grossulariata Linnaeus 1758 & mesophilous-hygrophilous & $\begin{array}{l}\text { Ribes rubrum, Ribes nigrum, Prunus spinosa, Crataegus, } \\
\text { Corylus }\end{array}$ & 6. \\
\hline
\end{tabular}




\begin{tabular}{|c|c|c|c|}
\hline Systematic list of recorded species & Habitat preference & Host plants & Locality \\
\hline 110. Ligdia adustata (Denis \& Schiffermüller 1775) & mesophilous & Euonymus europaeus, Euonymus verrucosa, Berberis & $\begin{array}{l}\text { 4., 6., 7., 8., 10., } \\
11 ., 13 ., 14 .\end{array}$ \\
\hline 111. Angerona prunaria Linnaeus 1758 & mesophilous-hygrophilous & $\begin{array}{l}\text { Prunus spinosa, Prunus domestica, Crataegus, Popu- } \\
\text { lus tremula, Salix caprea, Corylus, Vaccinium, Rubus, } \\
\text { Cytisus }\end{array}$ & $\begin{array}{l}\text { 3., 6., 7., 8., 11., } \\
\text { 12., 13., } 14 .\end{array}$ \\
\hline 112. Lomographa bimaculata (Fabricius 1775) & mesophilous-hygrophilous & $\begin{array}{l}\text { Prunus domestica, Crataegus, Prunus, Tilia, Quercus, } \\
\text { Prunus spinosa }\end{array}$ & 6., 7., $11 ., 14$. \\
\hline 113. Lomographa temerata (Denis \& Schiffermüller 1775) & mesophilous-hygrophilous & $\begin{array}{l}\text { Prunus domestica, Prunus spinosa, Malus, Rosa canina, } \\
\text { Salix Quercus }\end{array}$ & $\begin{array}{l}\text { 6., } 8 ., 11 ., 12 ., \\
14 .\end{array}$ \\
\hline 114. Agriopis marginaria Fabricius 1776 & mesophilous & Quercus, Fagus, Populus tremula & 1., $6 ., 14$. \\
\hline 115. Apocheima hispidaria (Denis \& Schiffermüller 1775) & mesophilous & Quercus, Salix, Betula, Carpinus, Alnus, Fagus, Ulmus & $\begin{array}{l}\text { 1., } 2 ., 3 ., 7 ., \\
8 ., 14 .\end{array}$ \\
\hline 116. Biston betularia (Linnaeus 1758) & mesophilous & Quercus, Betula, Ulmus, Fagus, Salix, Arbores fructiferae & 4. \\
\hline 117. Biston strataria (Hufnagel 1767) & mesophilous & $\begin{array}{l}\text { Quercus, Tilia, Populus, Salix, Betula, Alnus, Ulmus, } \\
\text { Acer, Arbores fructiferae, Corylus }\end{array}$ & 10., 11. \\
\hline 118. Erannis defoliaria (Clerck 1759) & mesophilous & $\begin{array}{l}\text { Quercus, Fagus, Carpinus, Tilia, Ulmus, Betula, Arbores } \\
\text { fructiferae }\end{array}$ & 3. \\
\hline 119. Lycia hirtaria (Clerck 1759) & mesophilous & $\begin{array}{l}\text { Ulmus, Betula, Fraxinus, Quercus, Populus, Tilia, Salix, } \\
\text { Crataegus, Prunus domestica, Robinia pseudacacia, } \\
\text { Arbores fructiferae }\end{array}$ & $\begin{array}{l}\text { 1., 2., 3., 4., 7., } \\
\text { 8., } 10 ., 11 ., 14 .\end{array}$ \\
\hline 120. Phigalia pilosaria (Denis \& Schiffermüller 1775) & mesophilous & $\begin{array}{l}\text { Quercus, Ulmus, Tilia, Prunus spinosa, Populus tremula, } \\
\text { Acer, Pyrus, Crataegus, Betula, Carpinus, Corylus }\end{array}$ & 1. \\
\hline 121. Ascotis selenaria Denis \& Schiffermüller 1775 & xerothermophilous & $\begin{array}{l}\text { Artemisia campestris, Sambucus nigra, Rubus idaeus, } \\
\text { Cytisus scoparius, Rosaceae }\end{array}$ & 3., 5 . \\
\hline $\begin{array}{l}\text { 122. Ectropis crepuscularia (Denis \& Schiffermüller } \\
1775 \text { ) }\end{array}$ & mesophilous & $\begin{array}{l}\text { Quercus, Betula, Salix, Ulmus, Populus, Alnus, Prunus } \\
\text { domestica }\end{array}$ & $\begin{array}{l}\text { 1., } 2 ., 3 ., 4 ., 6 . \\
8 ., 10 .\end{array}$ \\
\hline 123. Ematurga atomaria (Linnaeus 1758) & mesophilous-hygrophilous & $\begin{array}{l}\text { Calluna, Cytisus scoparius, Artemisia, Coronilla, Lotus } \\
\text { corniculatus, Scabiosa, Trifolium }\end{array}$ & 2., 3., 8., 11., 13 . \\
\hline 124. Hypomecis punctinalis (Scopoli 1763) & mesophilous & $\begin{array}{l}\text { Quercus, Betula, Prunus, Tilia, Populus, Salix, Prunus } \\
\text { spinosa, Tilia, Alnus }\end{array}$ & 3., 6., 11., 12. \\
\hline 125. Hypomecis roboraria (Denis \& Schiffermüller 1775) & mesophilous & Quercus, Fagus, Salix, Betula & 7. \\
\hline 126. Menophra abruptaria (Thunberg 1792) & $\begin{array}{l}\text { mesophilous-xerother- } \\
\text { mophilous }\end{array}$ & $\begin{array}{l}\text { Ligustrum vulgare, Syringa vulgaris, Clematis, Ulmus, } \\
\text { Quercus, Ribes rubrum, Prunus domestica, Cytisus, Tilia, } \\
\text { Jasminum, Arbutus unedo, Rosa canina }\end{array}$ & 1. \\
\hline 127. Nychiodes obscuraria (de Villers, 1789) & xerothermophilous & Prunus spinosa, Prunus, Genista, Ulex & 5. \\
\hline 128. Peribatodes correptaria (Zeller 1847) & mesophilous & Cupressus & 11. \\
\hline $\begin{array}{l}\text { 129. Peribatodes rhomboidaria (Denis \& Schiffermüller } \\
\text { 1775) }\end{array}$ & mesophilous & $\begin{array}{l}\text { Prunus spinosa, Crataegus, Frangula alnus, Ligustrum } \\
\text { vulgare, Clematis vitalba, Arbores fructiferae, Hedera } \\
\text { helix, Quercus, Fraxinus, Rubus, Lonicera, Rosa canina, } \\
\text { Clematis, Ligustrum, Prunus domestica }\end{array}$ & $\begin{array}{l}3 ., 5 ., 6 ., 9 ., 11 ., \\
12 ., 14 .\end{array}$ \\
\hline 130. Synopsia sociaria (Hübner, 1799) & $\begin{array}{l}\text { mesophilous-xerother- } \\
\text { mophilous }\end{array}$ & $\begin{array}{l}\text { Artemisia, Artemisia campestris, Thymus, Plantago, } \\
\text { Hippophae rhamnoides, Prunus spinosa, Quercus, Lavan- } \\
\text { dula, Centaurea, Tamarix, Lotus corniculatus }\end{array}$ & 6. \\
\hline 131. Cabera exanthemata (Scopoli 1763) & mesophilous & $\begin{array}{l}\text { Salix, Populus tremula, Alnus glutinosa, Betula, Corylus } \\
\text { avellana }\end{array}$ & $\begin{array}{l}\text { 6., 7., 11., 14., } \\
13 .\end{array}$ \\
\hline 132. Campaea margaritaria (Linnaeus 1761) & mesophilous & Fagus, Quercus, Carpinus, Betula, Salix caprea, Prunus & 1., 3., 8., 13., 14, \\
\hline 133. Lomaspilis marginata (Linnaeus 1758) & mesophilous-hygrophilous & Salix caprea & $6 ., 14$. \\
\hline 134. Stegania cararia (Hübner 1790) & mesophilous-hygrophilous & Populus, Populus tremula, Alnus glutinosa & $6 ., 14$. \\
\hline 135. Stegania trimaculata (de Villers 1789) & mesophilous-hygrophilous & Populus & 3., $4 ., 6$. \\
\hline 136. Colotois pennaria (Linnaeus 1761) & mesophilous & $\begin{array}{l}\text { Quercus, Carpinus, Salix caprea, Tilia, Betula, Arbores } \\
\text { fructiferae }\end{array}$ & 3. \\
\hline 137. Apeira syringaria Linnaeus 1758 & mesophilous & $\begin{array}{l}\text { Lonicera, Syringa vulgaris, Ligustrum vulgare, Fraxinus } \\
\text { excelsior, Symphoricarpos albus }\end{array}$ & 14. \\
\hline 138. Ennomos autumnaria (Werneburg 1859) & mesophilous & Tilia, Betula, Alnus glutinosa, Quercus, Salix & 13. \\
\hline 139. Ennomos fuscantaria (Haworth, 1809) & mesophilous-hygrophilous & Fraxinus excelsior & 5. \\
\hline 140. Ennomos quercaria (Hübner 1813) & xerothermophilous & Quercus robur, Quercus ilex & $6 ., 13$. \\
\hline 141. Pseudopanthera macularia (Linnaeus, 1758) & mesophilous & $\begin{array}{l}\text { Stachys sylvatica, Lamium, Mentha, Teucrium chamae- } \\
\text { drys }\end{array}$ & 3., 5., 14. \\
\hline 142. Selenia dentaria (Fabricius 1775) & mesophilous & $\begin{array}{l}\text { Betula, Alnus incana, Rubus idaeus, Filipendula ulmaria, } \\
\text { Sorbus aucuparia, Prunus padus, Tilia, Acer platanoides, } \\
\text { Rhamnus, Calluna vulgaris, Vaccinium, Ledum palustre }\end{array}$ & 1., $10 ., 11$. \\
\hline 143. Selenia lunularia (Hübner 1788) & $\begin{array}{l}\text { mesophilous-xerother- } \\
\text { mophilous }\end{array}$ & Alnus incana, Quercus, Rosa, Prunus, Fraxinus excelsior & $3 ., 11$. \\
\hline
\end{tabular}




\begin{tabular}{|c|c|c|c|}
\hline Systematic list of recorded species & Habitat preference & Host plants & Locality \\
\hline 144. Selenia tetralunaria (Hufnagel 1767) & mesophilous & Quercus, Tilia, Betula, Corylus, Salix, Alnus & 1. \\
\hline 145. Epione repandaria (Hufnagel 1767) & mesophilous-hygrophilous & Salix, Populus tremula & 3., $5 ., 14$. \\
\hline 146. Opisthograptis luteolata (Linnaeus 1758) & mesophilous & $\begin{array}{l}\text { Crataegus, Prunus spinosa, Sorbus, Lonicera, Salix, } \\
\text { Sorbus }\end{array}$ & 1., $3 ., 9$. \\
\hline 147. Crocallis tusciaria (Borkhausen 1793) & $\begin{array}{l}\text { mesophilous-xerother- } \\
\text { mophilous }\end{array}$ & $\begin{array}{l}\text { Prunus spinosa, Clematis vitalba, Frangula alnus, Cra- } \\
\text { taegus, Berberis }\end{array}$ & 4. \\
\hline 148. Plagodis dolabraria (Linnaeus 1767) & mesophilous & Quercus, Tilia, Betula, Sambucus & 11. \\
\hline 149. Plagodis pulveraria (Linnaeus 1758) & mesophilous & Rubus Rubus plicatus Salix Corylus Alnus Quercus Betula & $1 ., 14$ \\
\hline 150. Chiasmia clathrata (Linnaeus 1758) & $\begin{array}{l}\text { mesophilous-xerother- } \\
\text { mophilous }\end{array}$ & Trifolium, Medicago & 1., 2., 5., 10., 14. \\
\hline 151. Heliomata glarearia (Denis \& Schiffermüller 1775) & xerothermophilous & $\begin{array}{l}\text { Medicago lupulina, Trifolium, Lathyrus pratensis, Hippo- } \\
\text { crepis comosa }\end{array}$ & 11., 11. \\
\hline 152. Isturgia arenacearia (Denis \& Schiffermüller 1775) & xerothermophilous & Coronilla varia & 1., 2., 3., 11., 14. \\
\hline 153. Macaria alternata (Denis \& Schiffermüller 1775) & mesophilous & $\begin{array}{l}\text { Salix caprea, Quercus, Alnus, Prunus spinosa, Prunus } \\
\text { padus }\end{array}$ & 1., 6., 7., 8., 14. \\
\hline 154. Macaria liturata (Clerck 1759) & $\begin{array}{l}\text { mesophilous-xerother- } \\
\text { mophilous }\end{array}$ & Picea abies, Pinus sylvestris, Pinus strobus, Abies alba & 11., 14. \\
\hline 155. Ourapteryx sambucaria (Linnaeus 1758) & mesophilous & $\begin{array}{l}\text { Sambucus nigra, Clematis vitalba, Lonicera, Syringa, } \\
\text { Prunus spinosa, Hedera helix }\end{array}$ & 13. \\
\hline 156. Theria rupicapraria (Denis \& Schiffermüller, 1775) & $\begin{array}{l}\text { mesophilous-xerother- } \\
\text { mophilous }\end{array}$ & Prunus spinosa, Crataegus, Prunus domestica & 14. \\
\hline 157. Comibaena bajularia (Denis \& Schiffermüller 1775) & $\begin{array}{l}\text { mesophilous-xerother- } \\
\text { mophilous }\end{array}$ & Quercus & 14. \\
\hline 158. Hemithea aestivaria (Hübner 1789) & mesophilous & $\begin{array}{l}\text { Quercus, Prunus padus, Salix caprea, Prunus spinosa, } \\
\text { Betula, Corylus, Rhamnus, Ribes rubrum, Sorbus au- } \\
\text { cuparia, Tilia }\end{array}$ & 3., 13., 14. \\
\hline 159. Jodis lactearia (Linnaeus 1758) & mesophilous-hygrophilous & Corylus, Quercus, Betula, Vaccinium myrtillus & 6., 7., 8., 11., 13. \\
\hline 160. Aplasta ononaria (Fuessly, 1783) & xerothermophilous & Ononis spinosa, Ononis repens & 7., 8. \\
\hline 161. Cataclysme riguata (Hübner, 1813) & xerothermophilous & Galium verum, Galium mollugo, Galium glaucum & 7., 8. \\
\hline 162. Chloroclysta siterata (Hufnagel 1767) & mesophilous & Tilia, Quercus, Acer, Prunus padus, Arbores fructiferae & 1. \\
\hline 163. Chloroclystis v-ata (Haworth, 1809) & mesophilous-hygrophilous & $\begin{array}{l}\text { Eupatorium cannabinum, Origanum vulgare, Sambucus } \\
\text { nigra, Clematis vitalba, Angelica sylvestris, Achillea }\end{array}$ & 9. \\
\hline 164. Colostygia pectinataria (Knoch 1781) & mesophilous-hygrophilous & Galium, Lamium, Urtica & 3., 4., 6., 9 . \\
\hline 165. Cosmorhoe ocellata (Linnaeus 1758) & mesophilous-hygrophilous & Galium mollugo, Galium verum & 12. \\
\hline 166. Plemyria rubiginata (Denis \& Schiffermüller 1775) & mesophilous-hygrophilous & Alnus glutinosa, Alnus incana & 3., 11. \\
\hline 167. Eupithecia abbreviata Stephens, 1831 & xerothermophilous & Quercus & 2., 14. \\
\hline 168. Eupithecia insigniata (Hübner 1790) & xerothermophilous & $\begin{array}{l}\text { Malus sylvestris, Malus domestica, Crataegus, Prunus } \\
\text { spinosa, Pyrus }\end{array}$ & 8. \\
\hline 169. Eupithecia irriguata (Hübner, 1813) & xerothermophilous & Quercus & 1., 2., 4., 14. \\
\hline 170. Anticlea derivata Denis \& Schiffermüller 1775 & xerothermophilous & Rosaceae & 6., 8., 13. \\
\hline 171. Earophila badiata (Denis \& Schiffermüller 1775) & mesophilous & Rosaceae & 1., 6., 7., 8., 14. \\
\hline 172. Horisme tersata (Denis \& Schiffermüller 1775) & xerothermophilous & Clematis vitalba & 3., 8., $11 ., 14$. \\
\hline 173. Horisme vitalbata (Denis \& Schiffermüller 1775) & mesophilous-hygrophilous & Clematis vitalba & 9., 11., 13., 14. \\
\hline 174. Melanthia procellata (Denis \& Schiffermüller 1775) & mesophilous & Clematis vitalba & 5., 6., 8., 12., 14. \\
\hline 175. Epirrita dilutata (Denis \& Schiffermüller, 1775) & mesophilous-hygrophilous & $\begin{array}{l}\text { Quercus, Betula, Alnus, Fagus, Arbores fructiferae, Acer, } \\
\text { Corylus, Crataegus, Fraxinus, Ulmus }\end{array}$ & 6. \\
\hline 176. Philereme vetulata (Denis \& Schiffermüller, 1775) & $\begin{array}{l}\text { mesophilous-xerother- } \\
\text { mophilous }\end{array}$ & Rhamnus catharticus, Frangula alnus & 6. \\
\hline 177. Triphosa dubitata (Linnaeus 1758) & $\begin{array}{l}\text { mesophilous-xerother- } \\
\text { mophilous }\end{array}$ & Rhamnus catharticus & $6 ., 11$. \\
\hline 178. Acasis viretata (Hübner 1799) & mesophilous & $\begin{array}{l}\text { Rhamnus catharticus, Frangula alnus, Ligustrum vulgare, } \\
\text { Cornus sanguinea, Crataegus, Spiraea, Sorbus aucuparia }\end{array}$ & 8. \\
\hline 179. Camptogramma bilineata (Linnaeus 1758) & mesophilous & Galium, Rumex, Potentilla, Urtica, Ononis, Viola canina & 4. \\
\hline 180. Catarhoe cuculata (Hufnagel 1767) & mesophilous-hygrophilous & Galium mollugo, Galium verum & 14. \\
\hline 181. Catarhoe rubidata (Denis \& Schiffermüller 1775) & mesophilous & Galium mollugo, Galium verum, Galium odoratum & 12. \\
\hline 182. Costaconvexa polygrammata (Borkhausen 1794) & hygrophilous & $\begin{array}{l}\text { Galium mollugo, Galium palustre, Galium verum, Galium } \\
\text { saxatile }\end{array}$ & $1 ., 12$ \\
\hline
\end{tabular}




\begin{tabular}{|c|c|c|c|}
\hline Systematic list of recorded species & Habitat preference & Host plants & Locality \\
\hline 183. Nycterosea obstipata (Fabricius, 1794) & xerothermophilous & $\begin{array}{l}\text { Galium mollugo, Rumex, Convolvulus, Senecio, Anthemis, } \\
\text { Alyssum, Anthemis, Eupatorium, Nasturtium }\end{array}$ & 6. \\
\hline 184. Epirrhoe alternata (Müller 1764) & mesophilous-hygrophilous & Galium mollugo & 2., $11 ., 14$. \\
\hline 185. Scotopteryx luridata (Hufnagel 1767) & mesophilous & Cytisus scoparius, Genista, Ulex & $1 ., 11$. \\
\hline 186. Xanthorhoe ferrugata (Clerck 1759) & mesophilous-hygrophilous & Galium, Stellaria, Campanula & $\begin{array}{l}\text { 6., 7., 11., 13., } \\
14 .\end{array}$ \\
\hline 187. Xanthorhoe fluctuata (Linnaeus 1758) & mesophilous & Aster sedifolius, Sisymbrium, Alyssum, Alliaria, Brassica & 11. \\
\hline 188. Cyclophora albiocellaria (Hübner 1789) & xerothermophilous & Acer & 1. \\
\hline 189. Cyclophora annularia (Fabricius 1775) & mesophilous & Acer & $\begin{array}{l}\text { 6., 7., 8., 11., } \\
12 ., 13 ., 14 .\end{array}$ \\
\hline 190. Cyclophora pendularia (Clerck 1759) & hygrophilous & Salix caprea & 5., 7., 8., 10., 13. \\
\hline 191. Cyclophora puppillaria (Hübner 1799) & xerothermophilous & Quercus & 1. \\
\hline 192. Cyclophora suppunctaria (Zeller 1847) & xerothermophilous & $\begin{array}{l}\text { Quercus pubescens, Quercus robur, Quercus pyrenaica, } \\
\text { Quercus }\end{array}$ & 11. \\
\hline 193. Idaea aversata (Linnaeus 1758) & mesophilous & Polygonum & $1 ., 3$. \\
\hline 194. Idaea degeneraria (Hübner 1799) & mesophilous & Achillea, Scabiosa, Convolvulus, Frangula alnus & 3., 11., 13., 14. \\
\hline 195. Idaea moniliata (Denis \& Schiffermüller 1775) & xerothermophilous & Vicia, Leontodon, Myosotis & 1. \\
\hline 196. Idaea muricata (Hufnagel 1767) & hygrotermophilous & Potentilla palustris, Polygonum, Hypericum, Galium & $5 ., 14$. \\
\hline 197. Idaea politaria (Hubner 1799) & xerothermophilous & unknown & 1. \\
\hline 198. Idaea seriata (Schrank 1802) & $\begin{array}{l}\text { mesophilous-xerother- } \\
\text { mophilous }\end{array}$ & Lichens, Hedera helix & 1. \\
\hline 199. Rhodometra sacraria (Linnaeus 1767) & xerothermophilous & Polygonum, Taraxacum officinale, Rumex & 9. \\
\hline 200. Rhodostrophia vibicaria (Clerck 1759) & $\begin{array}{l}\text { mesophilous-xerother- } \\
\text { mophilous }\end{array}$ & $\begin{array}{l}\text { Calluna vulgaris, Cytisus scoparius, Coronilla varia, } \\
\text { Genista }\end{array}$ & 11. \\
\hline 201. Scopula imitaria (Hübner 1799) & xerothermophilous & Ligustrum & 6. \\
\hline 202. Scopula ornata (Scopoli 1763) & xerothermophilous & $\begin{array}{l}\text { Thymus, Origanum vulgare, Achillea millefolium, Men- } \\
\text { tha, Veronica }\end{array}$ & 2. \\
\hline 203. Scopula rubiginata (Hufnagel 1767) & xerothermophilous & $\begin{array}{l}\text { Polygonum aviculare, Thymus, Trifolium, Lotus, Convol- } \\
\text { vulus, Taraxacum officinale }\end{array}$ & 14. \\
\hline 204. Timandra comae Schmidt 1931 & mesophilous-hygrophilous & Rumex, Polygonum & 3., $6 ., 9$. \\
\hline \multicolumn{4}{|l|}{ Hepialidae } \\
\hline 205. Triodia sylvina (Linnaeus 1761) & $\begin{array}{l}\text { mesophilous-xerother- } \\
\text { mophilous }\end{array}$ & Malva & $6 ., 9$. \\
\hline \multicolumn{4}{|l|}{ Incurvariidae } \\
\hline 206. Incurvaria pectinea Haworth 1828 & mesophilous & $\begin{array}{l}\text { Alnus glutinosa, Betula pendula, Betula pubescens, } \\
\text { Corylus avellana, Carpinus }\end{array}$ & 8. \\
\hline \multicolumn{4}{|l|}{ Lasiocampidae } \\
\hline 207. Lasiocampa quercus (Linnaeus 1758) & mesophilous & Quercus, Salix, Rubus, Calluna vulgaris, Vaccinium & $3 ., 11$. \\
\hline 208. Macrothylacia rubi (Linnaeus, 1758) & mesophilous & $\begin{array}{l}\text { Calluna vulgaris, Rubus plicatus, Rubus, Quercus, Fra- } \\
\text { garia, Trifolium, Medicago }\end{array}$ & 9. \\
\hline 209. Gastropacha quercifolia (Linnaeus 1758) & mesophilous & $\begin{array}{l}\text { Salix caprea, Prunus spinosa, Corylus, Frangula alnus, } \\
\text { Sorbus aucuparia, Crataegus }\end{array}$ & $2 ., 3 ., 6 ., 14$ \\
\hline 210. Poecilocampa populi (Linnaeus 1758) & mesophilous & Populus, Salix, Betula, Quercus, Malus, Prunus, Pyrus & 4., 6. \\
\hline \multicolumn{4}{|l|}{ Limacodidae } \\
\hline 211. Apoda limacodes Hufnagel 1766 & mesophilous & Fagus, Quercus, Carpinus & $1 ., 13$. \\
\hline 212. Heterogenea asella (Denis \& Schiffermüller 1775) & mesophilous & $\begin{array}{l}\text { Quercus, Fagus, Carpinus, Acer, Prunus, Tilia, Ulmus, } \\
\text { Betula }\end{array}$ & 14. \\
\hline \multicolumn{4}{|l|}{ Noctuidae } \\
\hline 213. Acontia trabealis (Scopoli 1763) & xerothermophilous & Convolvulus arvensis, Calystegia sepium & $\begin{array}{l}2 ., 3 ., 6 ., 11 . \\
13 ., 14 .\end{array}$ \\
\hline 214. Aedia leucomelas (Linnaeus 1758) & thermophilous & Convolvulus, Calystegia sepium & $6 ., 9$. \\
\hline 215. Acronicta auricoma (Denis \& Schiffermüller 1775) & mesophilous & Betula, Rubus, Salix, Populus & $5 ., 7 ., 14$. \\
\hline 216. Acronicta rumicis (Linnaeus 1758) & mesophilous & Rumex, Salix, Crataegus, Plantago & 3. \\
\hline 217. Acronicta strigosa (Denis \& Schiffermüller 1775) & mesophilous-hygrophilous & Crataegus, Prunus, Sorbus, Rhamnus, Prunus padus & $\begin{array}{l}\text { 4., 6., 7., 10., } \\
11 ., 13 .\end{array}$ \\
\hline
\end{tabular}




\begin{tabular}{|c|c|c|c|}
\hline Systematic list of recorded species & Habitat preference & Host plants & Locality \\
\hline 218. Actronicta aceris (Linnaeus 1758) & mesophilous & Acer platanoides, Aesculus, Populus, Salix, Ulmus & 6. \\
\hline 219. Craniophora ligustri (Denis \& Schiffermüller 1775) & mesophilous & Fraxinus, Ligustrum vulgare, Syringa & $\begin{array}{l}\text { 2., 3., 4., 6., 7., } \\
\text { 8., 11., } 14 .\end{array}$ \\
\hline $\begin{array}{l}\text { 220. Subacronicta megacephala (Denis \& Schiffermüller } \\
1775 \text { ) }\end{array}$ & mesophilous-hygrophilous & Alnus, Populus, Salix & 2., 3., 6., 7. \\
\hline 221. Amphipyra pyramidea (Linnaeus 1758) & mesophilous & $\begin{array}{l}\text { Quercus, Ulmus, Salix, Tilia, Crataegus, Populus, Carpi- } \\
\text { nus, Syringa }\end{array}$ & 3., 9., 14. \\
\hline 222. Allophyes oxyacanthae (Linnaeus 1758) & mesophilous & $\begin{array}{l}\text { Crataegus, Prunus cerasus, Malus, Prunus, Pyrus, } \\
\text { Arbores fructiferae }\end{array}$ & 3. \\
\hline 223. Asteroscopus sphinx (Hufnagel 1766) & mesophilous & $\begin{array}{l}\text { Tilia, Quercus, Fagus, Carpinus, Salix, Populus, Cratae- } \\
\text { gus, Corylus avellana, Fraxinus excelsior }\end{array}$ & 3. \\
\hline 224. Brachionycha nubeculosa (Esper 1785) & mesophilous-hygrophilous & $\begin{array}{l}\text { Betula, Salix, Populus tremula, Prunus padus, Lonicera } \\
\text { xyloseteum, Tilia, Frangula alnus, Quercus, Ulmus }\end{array}$ & 7., 10. \\
\hline 225. Meganephria bimaculosa (Linnaeus 1767) & mesophilous & Ulmus, Prunus spinosa, Quercus & 2., 3 . \\
\hline 226. Valeria oleagina (Denis \& Schiffermüller 1775) & xerothermophilous & Prunus, Crataegus & 2., 3., $11 ., 14$. \\
\hline 227. Laspeyria flexula (Denis \& Schiffermüller 1775) & mesophilous & lichens & 3., 4., $13 ., 14$. \\
\hline 228. Cryphia algae (Fabricius 1775) & mesophilous-hygrophilous & lichens & 9., 14. \\
\hline 229. Eucarta amethystina (Hübner 1803) & mesophilous-hygrophilous & Daucus carota, Peucedanum, Petroselinum, Silene & 3., 6., $11 ., 14$. \\
\hline 230. Diloba caeruleocephala (Linnaeus 1758) & mesophilous & $\begin{array}{l}\text { Crataegus, Corylus, Malus, Prunus, Populus, Quercus, } \\
\text { Salix, Arbores fructiferae }\end{array}$ & 3. \\
\hline 231. Callopistria latreillei (Duponchel 1827) & mesophilous-hygrophilous & $\begin{array}{l}\text { Asplenium ceterach, Cochlearia officinalis, Adiantum } \\
\text { capillus-veneris }\end{array}$ & 2., 6. \\
\hline 232. Deltote bankiana (Fabricius 1775 ) & mesophilous-hygrophilous & $\begin{array}{l}\text { Poa annua, Poa pratensis, Carex, Calamagrostis, Cy- } \\
\text { perus }\end{array}$ & 3., 11. \\
\hline 233. Deltote pygarga (Hufnagel 1766) & mesophilous-hygrophilous & $\begin{array}{l}\text { Rubus idaeus, Lonicera, Molinia caerulea, Calamagros- } \\
\text { tis, Brachypodium, Dactylis }\end{array}$ & $\begin{array}{l}\text { 3., 4., 5., 8., 11., } \\
\text { 13., } 14 .\end{array}$ \\
\hline 234. Eutelia adulatrix (Hübner 1813) & xerothermophilous & Cotinus coggygria, Ligustrum vulgare & 13. \\
\hline 235. Helicoverpa armigera (Hübner 1808) & xerothermophilous & Reseda, Solanaceae, Nicotiana & 9. \\
\hline 236. Tyta luctuosa (Denis \& Schiffermüller 1775) & xerothermophilous & Convolvulus arvensis, Calystegia, Plantago & 2., 3., 9., 13., 14 \\
\hline 237. Chloantha hyperici (Denis \& Schiffermüller 1775) & xerothermophilous & Hypericum & 9. \\
\hline 238. Apamea scolopacina (Esper 1788) & mesophilous-hygrophilous & Briza, Melica, Milium, Poa & 13. \\
\hline 239. Luperina dumerilii (Duponchel 1826) & xerothermophilous & Gramineae & 2., $3 ., 9$. \\
\hline 240. Oligia latruncula (Denis \& Schiffermüller 1775) & mesophilous-hygrophilous & Gramineae & 3., 14 . \\
\hline 241. Oligia strigilis (Linnaeus 1758) & mesophilous-hygrophilous & Elymus, Dactylis, Poa & 6. \\
\hline 242. Oligia versicolor (Borkhausen 1792) & mesophilous-hygrophilous & Gramineae & 5. \\
\hline 243. Photedes morrisii (Dale 1837) & hygrophilous & Festuca & \\
\hline 244. Athetis hospes (Freyer 1831) & hygrotermophilous & various low-growing plants, Nicotiana & 2., 3., 14. \\
\hline 245. Caradrina clavipalpis Scopoli 1763 & xerothermophilous & Leontodon, Stellaria media, Lamium, Rumex acetosa & 6. \\
\hline 246. Charanyca trigrammica (Hufnagel 1766) & mesophilous & Leontodon, Plantago major, Plantago lanceolata & 3., 8., 13. \\
\hline 247. Hoplodrina ambigua (Denis \& Schiffermüller 1775) & $\begin{array}{l}\text { mesophilous-xerother- } \\
\text { mophilous }\end{array}$ & Leontodon, Plantago, Rumex, Lactuca & 1. \\
\hline 248. Hoplodrina blanda (Denis \& Schiffermüller 1775) & $\begin{array}{l}\text { mesophilous-xerother- } \\
\text { mophilous }\end{array}$ & $\begin{array}{l}\text { Plantago lanceolata, Rumex, Silene, Taraxacum, Sedum } \\
\text { purpureum }\end{array}$ & 14. \\
\hline 249. Dypterygia scabriuscula (Linnaeus 1758) & $\begin{array}{l}\text { mesophilous-xerother- } \\
\text { mophilous }\end{array}$ & $\begin{array}{l}\text { Polygala vulgaris, Rumex, Taraxacum, Polygonum } \\
\text { convolvulus }\end{array}$ & 3. \\
\hline 250. Mormo maura (Linnaeus 1758) & hygrophilous & $\begin{array}{l}\text { Fragaria, Lamium, Rumex, Betula, Crataegus, Salix, } \\
\text { Alnus }\end{array}$ & 3. \\
\hline 251. Polyphaenis sericata (Esper 1787) & xerothermophilous & Ligustrum, Lonicera, Cornus & 2., 3., 6., $11 ., 14$ \\
\hline 252. Elaphria venustula (Hübner 1790) & xerothermophilous & Alchemilla, Calluna, Genista, Potentilla, Rubus, Cytisus & 2., 5., 6., 13., 14 \\
\hline 253. Conisania luteago (Denis \& Schiffermüller 1775) & xerothermophilous & Silene, Dianthus, Lychnis & 3. \\
\hline 254. Lacanobia oleracea (Linnaeus 1758) & mesophilous & herbaceous plants, Atriplex & 3. \\
\hline 255. Leucania comma (Linnaeus 1761) & mesophilous-hygrophilous & $\begin{array}{l}\text { Festuca, Dactylis glomerata, Deschampsia flexuosa, } \\
\text { Gramineae }\end{array}$ & 14. \\
\hline 256. Leucania loreyi (Duponchel 1827) & xerothermophilous & Gramineae & 9. \\
\hline 257. Mythimna albipuncta (Denis \& Schiffermüller 1775) & mesophilous-hygrophilous & Gramineae, Salix caprea, Salix viminalis & 3., 4., 9. \\
\hline
\end{tabular}




\begin{tabular}{|c|c|c|c|}
\hline Systematic list of recorded species & Habitat preference & Host plants & Locality \\
\hline 258. Mythimna congrua (Hübner 1817) & xerothermophilous & Gramineae & 14. \\
\hline 259. Mythimna ferrago (Fabricius 1787) & mesophilous & Gramineae, Plantago, Taraxacum, Deschampsia flexuosa & 6. \\
\hline 260. Mythimna l-album (Linnaeus 1767) & mesophilous-hygrophilous & Gramineae, Bromus, Festuca & 10., $11 ., 14$. \\
\hline 261. Mythimna riparia (Rambur 1829) & mesophilous-hygrophilous & Calamagrostis & 4. \\
\hline 262. Mythimna scirpi (Duponchel 1836) & hygrophilous & unknown & 6., 14. \\
\hline 263. Mythimna turca (Linnaeus 1761) & mesophilous-hygrophilous & Dactylis glomerata, Poa nemoralis, Luzula, Gramineae & 2., 3 . \\
\hline 264. Mythimna vitellina (Hübner 1808) & xerothermophilous & Gramineae & 3., 8. \\
\hline 265. Agrotis exclamationis (Linnaeus 1758) & $\begin{array}{l}\text { mesophilous-xerother- } \\
\text { mophilous }\end{array}$ & $\begin{array}{l}\text { Plantago, Taraxacum officinale, Rumex acetosa, Artemi- } \\
\text { sia campestris, Plantago, Taraxacum officinale }\end{array}$ & $\begin{array}{l}\text { 3., 5., 6., 9., } \\
11 ., 14 .\end{array}$ \\
\hline 266. Agrotis ipsilon (Hufnagel 1766) & $\begin{array}{l}\text { mesophilous-xerother- } \\
\text { mophilous }\end{array}$ & Crataegus, Solanum tuberosum, Nicotiana, Beta & 3., 6., 8., 14 . \\
\hline 267. Agrotis segetum (Denis \& Schiffermüller 1775) & $\begin{array}{l}\text { mesophilous-xerother- } \\
\text { mophilous }\end{array}$ & Gramineae & 9. \\
\hline 268. Axylia putris (Linnaeus 1761) & mesophilous-hygrophilous & Rumex, Plantago, Stellaria, Polygonum, Galium, Atriplex & 3. \\
\hline 269. Cerastis rubricosa (Denis \& Schiffermüller 1775) & mesophilous-hygrophilous & $\begin{array}{l}\text { Fragaria, Galium, Lactuca, Plantago, Rumex, Salix, } \\
\text { Senecio, Taraxacum, Vaccinium, Alnus incana, Salix }\end{array}$ & 2. \\
\hline 270. Eugnorisma depuncta (Linnaeus 1761) & mesophilous & Galium, Lamium, Primula, Rumex, Urtica & 1. \\
\hline 271. Euxoa temera (Hübner 1808) & xerothermophilous & Beta vulgaris & 3., 7. \\
\hline 272. Noctua comes Hübner 1813 & $\begin{array}{l}\text { mesophilous-xerother- } \\
\text { mophilous }\end{array}$ & $\begin{array}{l}\text { Prunus spinosa, Crataegus, Salix, Calluna vulgaris, } \\
\text { Rumex }\end{array}$ & 1. \\
\hline 273. Noctua fimbriata (Schreber 1759) & mesophilous & $\begin{array}{l}\text { Primula, Rubus plicatus, Rubus, Taraxacum officinale, } \\
\text { Trifolium, Vitis vinifera }\end{array}$ & $\begin{array}{l}\text { 1., 3., 6., 8., 9., } \\
\text { 13., } 14 .\end{array}$ \\
\hline 274. Noctua janthina Denis \& Schiffermüller 1775 & mesophilous-hygrophilous & Crataegus, Primula, Rubus, Rumex, Ulmus, Urtica, Viola & $\begin{array}{l}\text { 1., 3., 6., 8., 9., } \\
\text { 13., } 14 .\end{array}$ \\
\hline 275. Noctua pronuba (Linnaeus 1758) & mesophilous-hygrophilous & $\begin{array}{l}\text { Gramineae, Brassica, Plantago major, Taraxacum offici- } \\
\text { nale, Hieracium }\end{array}$ & 3., 6 . \\
\hline 276. Ochropleura plecta (Linnaeus 1761) & mesophilous-hygrophilous & Galium, Senecio jacobaea & 5., 6., $13 ., 14$. \\
\hline 277. Spaelotis ravida (Denis \& Schiffermüller 1775) & xerothermophilous & Artemisia, Rumex, Taraxacum officinale, Cirsium & 11. \\
\hline 278. Xestia castanea (Esper 1798) & $\begin{array}{l}\text { mesophilous-xerother- } \\
\text { mophilous }\end{array}$ & Calluna vulgaris, Erica, Genista, Scoparia & 3. \\
\hline 279. Xestia c-nigrum (Linnaeus 1758) & mesophilous & Stellaria media, Trifolium, Epilobium angustifolium & $1 ., 3$. \\
\hline 280. Xestia stigmatica (Hübner 1813) & mesophilous & Lamium, Primula, Plantago, Stellaria, Taraxacum & 7. \\
\hline 281. Xestia xanthographa (Denis \& Schiffermüller 1775) & mesophilous-hygrophilous & Galium verum, Rumex, Plantago, Stellaria, Viola & 4., 9. \\
\hline 282. Anorthoa munda (Denis \& Schiffermüller 1775) & mesophilous & Carpinus, Fagus, Quercus, Prunus, Salix, Tilia, Ulmus & $\begin{array}{l}\text { 1., 3., 7., 8., 10., } \\
11 ., 14 .\end{array}$ \\
\hline 283. Egira conspicillaris (Linnaeus 1758) & mesophilous & Tilia, Acer, Cytisus, Rumex, Artemisia & 4., 7., 14. \\
\hline 284. Orthosia cerasi (Fabricius 1775) & xerothermophilous & Betula, Quercus, Salix, Ulmus & 2., 3., 8., 11., 14. \\
\hline 285. Orthosia cruda (Denis \& Schiffermüller 1775) & mesophilous & Quercus, Betula, Crataegus, Acer, Salix, Lonicera & 2., 3., 4., 10., 14. \\
\hline 286. Orthosia gothica (Linnaeus 1758) & mesophilous & $\begin{array}{l}\text { Tilia, Quercus, Populus, Betula, Alnus incana, Corylus } \\
\text { avellana, Prunus padus, Artemisia, Taraxacum officinale, } \\
\text { Prunus spinosa }\end{array}$ & $\begin{array}{l}\text { 1., 2., 7., 8., 10., } \\
\text { 11., } 14 .\end{array}$ \\
\hline 287. Orthosia incerta (Hufnagel 1766) & mesophilous & $\begin{array}{l}\text { Salix, Populus, Quercus, Betula, Vaccinium uliginosum, } \\
\text { Vaccinium myrtillus, Prunus spinosa }\end{array}$ & $\begin{array}{l}\text { 1., 2., 3., 4., 8., } \\
\text { 10., 11., } 14 .\end{array}$ \\
\hline 288. Orthosia miniosa (Denis \& Schiffermüller 1775) & mesophilous & Acer, Fagus, Betula, Populus, Quercus & 3. \\
\hline 289. Orthosia opima (Hübner 1809) & eurytopic & $\begin{array}{l}\text { Betula, Quercus, Salix, Alnus, Calluna, Vaccinium, } \\
\text { Trifolium }\end{array}$ & 2., 3., 11. \\
\hline 290. Panolis flammea (Denis \& Schiffermüller, 1775) & mesophilous & Pinus sylvestris, Picea & 9., 10. \\
\hline 291. Euplexa lucipara (Linnaeus 1758) & mesophilous & $\begin{array}{l}\text { Epilobium, Lamium, Urtica, Salix, Pteridium, Betula, } \\
\text { Salix }\end{array}$ & $6 ., 11$. \\
\hline 292. Spodoptera exigua (Hübner 1808) & xerothermophilous & $\begin{array}{l}\text { Asparagus, Beta vulgaris, Apium, Lactuca, Solanum } \\
\text { tuberosum, Lycopersicon lycopersicum, Gossypium, } \\
\text { Nicotiana }\end{array}$ & 9. \\
\hline 293. Agrochola lota (Clerck 1759) & mesophilous-hygrophilous & Salix, Populus, Alnus & 4. \\
\hline 294. Atethmia centrago (Haworth 1809) & mesophilous & Fraxinus & 2., 3., 4., 13. \\
\hline $\begin{array}{l}\text { 295. Conistra erythrocephala (Denis \& Schiffermüller } \\
1775 \text { ) }\end{array}$ & mesophilous & $\begin{array}{l}\text { Carpinus, Fagus, Quercus, Ulex, Fragaria, Galium, } \\
\text { Plantago }\end{array}$ & 2. \\
\hline 296. Conistra rubiginea (Denis \& Schiffermüller 1775) & mesophilous & Prunus, Rosa, Salix, Rubus, Fragaria, Rumex, Taraxacum & $1 ., 11$. \\
\hline
\end{tabular}




\begin{tabular}{|c|c|c|c|}
\hline Systematic list of recorded species & Habitat preference & Host plants & Locality \\
\hline 297. Conistra vaccinii (Linnaeus 1761) & mesophilous & $\begin{array}{l}\text { Populus, Quercus, Betula, Ulmus, Malus, Ribes, Rubus, } \\
\text { Prunus padus, Vaccinium }\end{array}$ & $\begin{array}{l}1 ., 3 ., 6 ., 8 . \\
11 ., 14 .\end{array}$ \\
\hline 298. Trigonophora flammea (Esper, 1785) & $\begin{array}{l}\text { mesophilous-xerother- } \\
\text { mophilous }\end{array}$ & $\begin{array}{l}\text { Ranunculus, Ligustrum vulgare, Fraxinus, Prunus, } \\
\text { Chamaecytisus }\end{array}$ & 7. \\
\hline 299. Cosmia affinis (Linnaeus 1767) & mesophilous-hygrophilous & Ulmus, Quercus & 1., 2., 3., 6., 14 . \\
\hline 300. Cosmia diffinis (Linnaeus 1767) & mesophilous-hygrophilous & Ulmus & 6. \\
\hline 301. Cosmia pyralina (Denis \& Schiffermüller 1775) & mesophilous & Betula, Populus, Quercus, Salix, Tilia, Ulmus, Pyrus & $6 ., 11 ., 13$. \\
\hline 302. Cosmia trapezina (Linnaeus 1758) & mesophilous & Quercus, Betula, Ulmus glabra, Corylus avellana, Prunus & $1 ., 6 ., 11 ., 14$ \\
\hline 303. Dryobotodes eremita (Fabricius 1775) & $\begin{array}{l}\text { mesophilous-xerother- } \\
\text { mophilous }\end{array}$ & Quercus & 1. \\
\hline 304. Eupsilia transversa (Hufnagel 1766) & mesophilous & $\begin{array}{l}\text { Betula, Corylus avellana, Quercus robur, Pyrus commu- } \\
\text { nis, Malus domestica }\end{array}$ & 1., $7 ., 14$. \\
\hline 305. Ipimorpha retusa (Linnaeus 1761) & mesophilous-hygrophilous & Alnus, Populus, Salix & 6. \\
\hline 306. Ipimorpha subtusa (Denis \& Schiffermüller 1775) & mesophilous-hygrophilous & Populus, Populus tremula, Malus, Pyrus, Salix & 6. \\
\hline 307. Lithophane ornitopus (Hufnagel 1766) & $\begin{array}{l}\text { mesophilous-xerother- } \\
\text { mophilous }\end{array}$ & Quercus, Populus, Prunus padus, Salix & 7. \\
\hline 308. Lithophane socia (Hufnagel 1766) & mesophilous & Fraxinus, Prunus, Quercus, Ulmus, Tilia & 7., $11 ., 14$. \\
\hline 309. Litophane semibrunnea (Haworth 1809) & $\begin{array}{l}\text { mesophilous-xerother- } \\
\text { mophilous }\end{array}$ & Fraxinus, Prunus, Quercus & 7. \\
\hline 310. Mesogona oxalina (Hübner 1803) & mesophilous-hygrophilous & Alnus, Populus, Salix, Quercus & 2. \\
\hline 311. Tiliacea sulphurago (Denis \& Schiffermüller 1775) & xerophilous & Acer, Betula, Fagus, Quercus, Salix, Tilia, Fraxinus & 1. \\
\hline 312. Colocasia coryli (Linnaeus 1758) & mesophilous & $\begin{array}{l}\text { Betula, Alnus incana, Corylus avellana, Quercus, Salix, } \\
\text { Populus, tremula, Malus domestica, Crataegus, Tilia, } \\
\text { Acer, Fagus sylvatica }\end{array}$ & $\begin{array}{l}3 ., 4 ., 6 ., 7 ., 8 ., \\
10 ., 11 ., 12 ., \\
13 ., 14 .\end{array}$ \\
\hline 313. Chrysodeixis chalcites (Esper 1789) & thermophilous & Echium, Apium, Rubus & 9. \\
\hline 314. Autographa gamma (Linnaeus 1758) & eurytopic & Urtica, Trifolium & $\begin{array}{l}\text { 2., 3., 4., 6., 7., } \\
\text { 8., 10., 11., 12., } \\
13 ., 14 .\end{array}$ \\
\hline 315. Macdunnoughia confusa (Stephens 1850) & thermophilous & Matricaria, Urtica dioica & 2., 3., 11. \\
\hline \multicolumn{4}{|l|}{ Nolidae } \\
\hline 316. Pseudoips prasinana (Linnaeus 1758) & mesophilous & Fagus, Quercus, Populus, Betula, Carpinus & $13 ., 14$. \\
\hline 317. Bena bicolorana (Fuessly, 1775) & mesophilous & Quercus robur, Fagus & 7., 9., 13. \\
\hline 318. Earias clorana (Linnaeus 1761) & mesophilous-hygrophilous & Salix viminalis, Salix & $6 ., 14$. \\
\hline 319. Nyctaeola revayana (Scopoli 1772) & mesophilous & Quercus, Salix, Populus & 13., 14. \\
\hline 320. Nyctaeola siculana (Fuchs 1899) & hygrophilous & Salix, Populus & $6 ., 7 ., 14$. \\
\hline 321. Nycteola asiatica (Krulikovsky 1904) & hygrophilous & Salix, Populus & 3. \\
\hline 322. Meganola albula (Denis \& Schiffermüller 1775) & hygrophilous & Mentha aquatica, Rubus, Rubus idaeus, Fragaria vesca & 6. \\
\hline 323. Meganola togatulalis (Hübner 1796) & mesophilous & Quercus, Prunus spinosa & 3. \\
\hline 324. Nola aerugula (Hübner 1793) & hygrophilous & $\begin{array}{l}\text { Fragaria, Potentilla, Rubus, Betula, Trifolium, Lotus, } \\
\text { Medicago }\end{array}$ & 4., 14. \\
\hline 325. Nola cicatricalis (Treitschke 1835) & mesophilous & lichens & $1 ., 3 ., 10 ., 14$. \\
\hline 326. Nola confusalis (Herrich-Schäffer 1847) & mesophilous & Quercus, Fagus, Carpinus, Tilia, Crataegus & $6 ., 8$. \\
\hline 327. Nola cucullatella (Linnaeus 1758) & xerothermophilous & $\begin{array}{l}\text { Prunus spinosa, Prunus domestica, Crataegus, Sorbus } \\
\text { aucuparia, Quercus }\end{array}$ & 14. \\
\hline \multicolumn{4}{|l|}{ Notodontidae } \\
\hline 328. Spatalia argentina (Denis \& Schiffermuller, 1775) & mesophilous & Quercus, Salix, Populus & 13. \\
\hline 329. Harpyia milhauseri (Fabricius, 1775) & mesophilous & Quercus, Fagus, Betula, Carpinus & $6 ., 13$. \\
\hline 330. Stauropus fagi (Linnaeus 1758) & mesophilous & $\begin{array}{l}\text { Fagus sylvatica, Quercus, Carpinus betulus, Tilia, Cory- } \\
\text { lus, Betula, Crataegus, Malus }\end{array}$ & $3 ., 6 ., 7$. \\
\hline 331. Dicranura ulmi (Denis \& Schiffermüller 1775) & mesophilous & Ulmus & 2., $11 ., 14$. \\
\hline 332. Furcula furcula (Clerck 1759) & mesophilous & Betula, Populus tremula, Salix caprea, Populus, Salix & $\begin{array}{l}3 ., 6 ., 7 ., 8 ., \\
10 ., 14 .\end{array}$ \\
\hline 333. Drymonia dodonaea (Denis \& Schiffermüller 1775) & mesophilous & Quercus, Fagus, Betula & 8. \\
\hline 334. Drymonia ruficornis (Hufnagel 1766) & xerothermophilous & Quercus & $\begin{array}{l}\text { 1., 3., 6., 11., } \\
12 ., 14 .\end{array}$ \\
\hline
\end{tabular}




\begin{tabular}{|c|c|c|c|}
\hline Systematic list of recorded species & Habitat preference & Host plants & Locality \\
\hline 335. Notodonta tritophus (Denis \& Schiffermüller 1775) & mesophilous-hygrophilous & Populus tremula, Populus, Salix, Betula & 3. \\
\hline 336. Pheosia tremula (Clerck 1759) & mesophilous & $\begin{array}{l}\text { Populus, Populus tremula, Populus canadensis, Salix, } \\
\text { Betula }\end{array}$ & 7. \\
\hline 337. Pterostoma palpina (Clerck 1759) & mesophilous & Populus, Salix, Populus tremula, Tilia, Alnus, Quercus & 3., $6 ., 7$. \\
\hline 338. Ptilodon capucina (Linnaeus 1758) & mesophilous & Betula, Alnus, Salix, Tilia, Quercus, Populus tremula & 8., 12., $13 ., 14$. \\
\hline 339. Ptilodon cucullina (Denis \& Schiffermüller 1775) & mesophilous & Acer campestre, Acer pseudoplatanus, Quercus, Ulmus & $\begin{array}{l}\text { 3., 6., 11., 12., } \\
\text { 13., } 14 .\end{array}$ \\
\hline 340. Peridea anceps (Goeze 1781) & mesophilous & Quercus, Betula, Fagus & 1., 6., 7., 11., 14. \\
\hline 341. Phalera bucephaloides (Ochsenheimer 1810) & xerothermophilous & Quercus & 1. \\
\hline 342. Clostera anastomosis (Linnaeus 1758) & mesophilous & Salix, Populus, Populus tremula & 2., 3 . \\
\hline 343. Clostera curtula (Linnaeus 1758) & mesophilous & Populus, Salix & 4., 7., 11. \\
\hline 344. Clostera pigra (Hufnagel 1766) & mesophilous & Salix aurita, Salix repens, Populus tremula, Populus & 3. \\
\hline \multicolumn{4}{|l|}{ Oecophoridae } \\
\hline 345. Harpella forficella (Scopoli 1763) & mesophilous & Quercus & 6. \\
\hline \multicolumn{4}{|l|}{ Peleopodidae } \\
\hline 346. Carcina quercana (Fabricius 1775) & mesophilous & $\begin{array}{l}\text { Quercus, Fagus, Pyrus, Malus, Castanea sativa, Acer } \\
\text { pseudoplatanus, Crataegus, Sorbus }\end{array}$ & 13. \\
\hline \multicolumn{4}{|l|}{ Psychidae } \\
\hline 347. Megalophanes viciella (Denis \& Schiffermüller 1775) & mesophilous & $\begin{array}{l}\text { Vicia, Rumex, Scirpus, Calluna vulgaris, Betula pubes- } \\
\text { cens, Stachys palustris, Vaccinium uliginosum }\end{array}$ & 14. \\
\hline 348. Sterrhopterix fusca (Haworth 1809) & mesophilous & $\begin{array}{l}\text { Gramineae, Quercus, Crataegus, Salix, Betula, Calluna } \\
\text { vulgaris, Erica }\end{array}$ & 8. \\
\hline \multicolumn{4}{|l|}{ Pterophoridae } \\
\hline 349. Amblyptilia acanthadactyla (Hübner 1813) & mesophilous & Stachys, Salvia, Euphrasia, Ononis, Chenopodium & 5., 11., 14., \\
\hline $\begin{array}{l}\text { 350. Cnaemidophorus rhododactyla (Denis \& Schiffer- } \\
\text { müller 1775) }\end{array}$ & mesophilous & Rosa canina & 11., 14. \\
\hline 351. Crombrugghia distans (Zeller 1847) & mesophilous & Crepis, Hieracium, Picris, Sonchus & 5. \\
\hline 352. Emmelina monodactyla (Linnaeus 1758) & xerothermophilous & Convolvulus arvensis, Calystegia, Atriplex & 9. \\
\hline 353. Pterophorus pentadactyla (Linnaeus 1758) & xerothermophilous & $\begin{array}{l}\text { Convolvulus arvensis, Prunus spinosa, Trifolium, Anemo- } \\
\text { ne, Calystegia sepium }\end{array}$ & 3., $6 ., 13$. \\
\hline \multicolumn{4}{|l|}{ Pyralidae } \\
\hline 354. Acrobasis tumidana (Denis \& Schiffermüller 1775) & mesophilous & Quercus & 6. \\
\hline 355. Euzophera pinguis (Haworth 1811) & mesophilous & Fraxinus excelsior & 2. \\
\hline 356. Homoeosoma sinuella (Fabricius 1794) & mesophilous & Plantago lanceolata & 3., 6., 14 . \\
\hline 357 Nephopterix angustella (Hübner 1796) & mesophilous & Euonymus europaeus & $1 ., 12$. \\
\hline 358. Oncocera semirubella (Scopoli 1763) & mesophilous & $\begin{array}{l}\text { Lotus corniculatus, Trifolium repens, Medicago sativa, } \\
\text { Ononis arvensis }\end{array}$ & 1., 3., $6 ., 14$ \\
\hline 359. Pempelia palumbella (Denis \& Schiffermüller 1775) & mesophilous & $\begin{array}{l}\text { Calluna vulgaris, Erica tetralix, Helianthemum, Thymus, } \\
\text { Polygala vulgaris }\end{array}$ & 11. \\
\hline 360. Phycita roborella (Denis \& Schiffermüller 1775) & mesophilous & Quercus & 1., 14. \\
\hline 361. Trachonitis cristella (Denis \& Schiffermüller 1775) & mesophilous & Prunus spinosa, Pyrus, Euonymus europaeus, Betula & 14. \\
\hline 362. Endotricha flammealis (Denis \& Schiffermüller 1775) & mesophilous & $\begin{array}{l}\text { Quercus spp., Salix spp., Lotus, Agrimonia, Vaccinum } \\
\text { spp. }\end{array}$ & $1 ., 6$. \\
\hline 363. Hypsopygia costalis (Fabricius 1775) & synanthropic & detritus & 3., 9., 14. \\
\hline 364. Hypsopygia glaucinalis (Linnaeus 1758) & mesophilous & detritus & 2. \\
\hline 365. Pyralis farinalis (Linnaeus 1758) & synanthropic & detritus & 4. \\
\hline 366. Pyralis regalis Denis \& Schiffermüller 1775 & mesophilous & Rosa, Quercus, Salix spp. & 1., 2., 3., 6., 14. \\
\hline 367. Stemmatophora brunnealis (Treitschke 1829) & xerothermophilous & $\begin{array}{l}\text { In silk tubes at base of low plants, including Helianthe- } \\
\text { mum, Globularia \& Epilobium }\end{array}$ & 3. \\
\hline \multicolumn{4}{|l|}{ Saturniidae } \\
\hline 368. Aglia tau (Linnaeus 1758) & mesophilous & Fagus & 11., 14. \\
\hline 369. Saturnia pavoniella (Scopoli 1763) & mesophilous & $\begin{array}{l}\text { Rubus, Prunus, Crataegus, Quercus, Carpinus, Betula, } \\
\text { Salix, Erica, Calluna, Filipendula }\end{array}$ & 2., 3 . \\
\hline
\end{tabular}




\begin{tabular}{|c|c|c|c|}
\hline Systematic list of recorded species & Habitat preference & Host plants & Locality \\
\hline 370. Saturnia pyri (Denis \& Schiffermüller 1775) & mesophilous & Malus, Pyrus, Prunus, Arbores fructiferae, Prunus avium & 7., 8., 11. \\
\hline \multicolumn{4}{|l|}{ Sphingidae } \\
\hline 371. Deilephila porcellus (Linnaeus 1758) & mesophilous & Galium mollugo, Galium verum & 6., 7. \\
\hline 372. Laothoe populi (Linnaeus 1758) & mesophilous-hygrophilous & $\begin{array}{l}\text { Populus tremula, Populus balsamifera, Populus alba, } \\
\text { Populus nigra }\end{array}$ & 3., 7. \\
\hline 373. Marumba quercus (Denis \& Schiffermüller, 1775) & xerothermophilous & Quercus & 6. \\
\hline 374. Mimas tiliae (Linnaeus 1758) & mesophilous & Tilia & 6., 8., 12. \\
\hline 375. Smerinthus ocellata (Linnaeus 1758) & mesophilous-hygrophilous & Salix & 6. \\
\hline 376. Argius convolvuli (Linnaeus 1758) & eurytopic & Convolvulus arvensis, Convolvulus, Calystegia sepium & 9. \\
\hline 377. Macroglossum stellatarum (Linnaeus, 1758) & eurytopic & Galium verum, Galium silvaticum, Galium mollugo & 4., $6 ., 7$. \\
\hline \multicolumn{4}{|l|}{ Torticidae } \\
\hline $\begin{array}{l}\text { 378. Ancylis mitterbacheriana (Denis \& Schiffermüller } \\
1775 \text { ) }\end{array}$ & xerothermophilous & Quercus, Fagus, Malus & 13. \\
\hline 379. Epiblema foenella (Linnaeus 1758) & xerothermophilous & Artemisia vulgaris & 2. \\
\hline 380. Eucosma cana (Haworth 1811) & xerothermophilous & Cirsium oleraceum, Carduus spp. & 11. \\
\hline 381. Notocelia cynosbatella (Linnaeus 1758) & eurytopic & $\begin{array}{l}\text { Rosa, Malus, Pyrus, Prunus, Crataegus, Carpinus, } \\
\text { quercus }\end{array}$ & 2., 4., 11. \\
\hline 382. Notocelia uddmanniana (Linnaeus 1758) & mesophilous & Rubus & 3., 5., 14. \\
\hline 383. Rhyacionia buoliana (Denis \& Schiffermüller 1775) & mesophilous & Pinus sylvesris & 14. \\
\hline 384. Cydia pomonella (Linnaeus 1758) & mesophilous & $\begin{array}{l}\text { Malus, Cydonia, Prunus persica, P. domestica, Sorbus } \\
\text { aucuparia, Armeniaca vulgaria, Castanea, Juglans }\end{array}$ & 14. \\
\hline 385. Celypha lacunana (Denis \& Schiffermüller 1775) & mesophilous & $\begin{array}{l}\text { Mentha, Artemisia, Inula, Chrysanthemum, Sanguisorba, } \\
\text { Ononis, Epilobium, Ranunculus, Caltha palustris, Fili- } \\
\text { pendula, Spiraea, Sorbus, Prunus cerasus, Fragaria, } \\
\text { Rubus, Ligustrum, Fagus, Urtica, Salix, Betula, Larix, } \\
\text { Abies alba, Pteridium aquilinum }\end{array}$ & 6. \\
\hline 386. Celypha striana (Denis \& Schiffermüller 1775) & mesophilous & Taraxacum oficinale, Plantago lanceolata & 6. \\
\hline 387. Hedya pruniana (Hübner 1799) & mesophilous & $\begin{array}{l}\text { Prunus spiniosa, P. divaricata, Crataegus, Rosa spp., } \\
\text { Sorbus, Corylus avellana, malus, Pyrus, Prunus }\end{array}$ & 6., $11 ., 14$. \\
\hline 388. Hedya salicella (Linnaeus 1758) & mesophilous-hygrophilous & Salix caprea, Populus tremula, P. nigra & 14. \\
\hline 389. Archips crataegana (Hübner 1799) & mesophilous & $\begin{array}{l}\text { Betula, Tilia, Crataegus, Pyrus, Salix, Populus, Prunus, } \\
\text { Quercus }\end{array}$ & 3. \\
\hline 390. Archips podana (Scopoli 1763) & mesophilous & $\begin{array}{l}\text { Corylus, Fagus, Malus, Pyrus, Rosa, ribes, Vaccinium, } \\
\text { Trifolium, Picea }\end{array}$ & 3., 8., 11. \\
\hline 391. Archips rosana (Linnaeus 1758) & mesophilous & Malus, Pyrus, Ligustrum & 5., $11 ., 14$. \\
\hline 392. Archips xylosteana (Linnaeus 1758) & mesophilous & Acer, Crataegus, Betula, Lonicera, Pyrus, Malus & 5., 14. \\
\hline 393. Syndemis musculana (Hübner 1799) & mesophilous & $\begin{array}{l}\text { Srbus, Rubus, Prunus, Padus, Crataegus, Betula, tilia, } \\
\text { salix, Quercus, Picea, Larix }\end{array}$ & $\begin{array}{l}\text { 4., 6., 8., 11., } \\
\text { 13., } 14 .\end{array}$ \\
\hline 394. Tortricodes alternella (Denis \& Schiffermüller 1775) & mesophilous & $\begin{array}{l}\text { Quercus, Carpinus, Betula, SAlix, Corylus, Crataegus, } \\
\text { Prunus }\end{array}$ & 14. \\
\hline 395. Agapeta zoegana (Linnaeus 1767) & mesophilous & $\begin{array}{l}\text { Scabiosa columbaria, Jurinea, Centaurea panniculata, } C \text {. } \\
\text { nigra, C. jacea }\end{array}$ & 3. \\
\hline 396. Acleris ferrugana (Denis \& Schiffermüller 1775) & mesophilous & Quercus, Fagus, Carpinus, Betula, Salix caprea & 6. \\
\hline 397. Acleris rhombana (Denis \& Schiffermüller 1775) & mesophilous & $\begin{array}{l}\text { Quercus, Corylus avellana, Crataegus, Prunus, Pyrus, } \\
\text { Rosa, Sorbus aucuparia }\end{array}$ & 3. \\
\hline 398. Aleimma loeflingiana (Linnaeus 1758) & mesophilous & Quercus & 11., 14. \\
\hline 399. Tortix viridana Linnaeus 1758 & xerothermophilous & Quercus robur, Q. pubescens, $Q$. petrarea & 4., 14. \\
\hline \multicolumn{4}{|l|}{ Yponomeutidae } \\
\hline 400. Yponomeuta cagnagella (Hübner 1813) & mesophilous & Euonymus europaeus, Frangula alnus & 6., 8. \\
\hline 401. Yponomeuta padella (Linnaeus 1758) & xerothermophilous & Prunus spinosa, Crataegus, Prunus & 14. \\
\hline \multicolumn{4}{|l|}{ Ypsolophidae } \\
\hline 402. Ypsolopha mucronella (Scopoli 1763) & mesophilous & Euonymus europaeus & 1., 3., 14. \\
\hline 403. Ypsolopha sequella (Clerck 1759) & mesophilous & Acer campestre, Acer pseudoplatanus & 1. \\
\hline
\end{tabular}

*Species identified on the basis of genitalia examination. 